\title{
Metamorphic Conditions of an Archean Core Complex in the Northern Wind River Range, Wyoming*
}

\author{
by Z. D. SHARP ${ }^{1}$ AND E. J. ESSENE ${ }^{2}$ \\ ${ }^{1}$ Institut de Minéralogie, Université de Lausanne, BFSH-2, CH-1015, Lausanne, Switzerland \\ ${ }^{2}$ Department of Geological Sciences, University of Michigan, Ann Arbor, Michigan \\ 48109-1063
}

(Received 6 March 1990; revised typescript accepted 11 July 1990)

\begin{abstract}
The Archean granulite-facies rocks of the northern Wind River Range consist of extensive granitic orthogneisses and migmatites hosting banded iron formations, amphibolites, metapelites, metabasites, ultramafites and quartzites. Quantitative pressure and temperature estimates from inclusions within garnet porphyroblasts are $815 \pm 50^{\circ} \mathrm{C}$ and $8 \pm 1 \mathrm{~kb}$ using equilibria buffered by the assemblages spinel-quartz-garnet-sillimanite and garnet-rutile-ilmenite-sillimanite-quartz Pressure-temperature estimates from the groundmass core assemblages of the banded iron formations and hornblende granulites are $750 \pm 50^{\circ} \mathrm{C}$ and $5 \cdot 5 \pm 1 \mathrm{~kb}$ using garnet-clinopyroxene, garnet-orthopyroxene, and twopyroxene thermometry, and geobarometers based on the assemblages garnet-quartz-plagioclaseorthopyroxene and orthopyroxene-olivine-quartz. Rim compositions of the matrix minerals indicate nearly isobaric cooling from the conditions of $750^{\circ} \mathrm{C}$ and $5.5 \mathrm{~kb}$ to $<600^{\circ} \mathrm{C}$ at $5 \mathrm{~kb}$. Taken together, the $P-T$ estimates from both the garnet inclusions and matrix assemblages are consistent with a clockwise $P-T-t$ path for this terrane.

Temperature estimates based on oxygen isotope thermometry in the banded iron formations vary systematically with the degree of visible late-stage deformation. There is no correlation between the isotopic temperature estimates and those from cation-based thermometers. The highest pressures and temperatures for the Wind River terrane are preserved by the inclusions in garnet porphyroblasts. The ability of these inclusions to preserve chemistries corresponding to higher pressures and temperatures is attributed to the combined effects of inclusion isolation and fixed inclusion volume within the garnet porphyroblasts. Cation-based thermometers in the groundmass preserve lower temperatures as a result of diffusional partial resetting. Isotopic thermometry will yield the lowest temperatures if there is even minor retrograde deformation.

Geothermobarometry for the northern Wind River Archean terrane is consistent with a tectonic regime of doubly thickened crust. Peak metamorphic conditions preserved in the cores of the garnets are compatible with deep burial during the early stages of tectonism. Rapid to intermediate uplift due to erosion of the upper plate could explain the nearly isothermal decompression from 8.0 to $5.5 \mathrm{~kb}$. The later, nearly isobaric, cooling path indicated by the rim compositions of the matrix minerals is consistent with relaxation of the elevated geotherm.
\end{abstract}

\section{INTRODUCTION}

The study of Archean high-grade metamorphic terranes is one of the most direct methods of evaluating conditions of early continental lower-crustal formation. Variations in the pressure-temperature conditions during a metamorphic event are related to the tectonic and heat-generating processes that were active during the metamorphism, and estimates of pressures and temperatures over the course of the metamorphic event may be used to place

* Contribution No. 472 from the Mineralogical Laboratory, The University of Michigan 
limits on the associated tectonic processes (e.g., Tracy, 1982; Spear \& Selverstone, 1983; England \& Thompson, 1984; Spear \& Rumble, 1986; Bohlen, 1987). In most granulite-facies terranes, chemical and textural information from the prograde history of the metamorphism is lost during the subsequent annealing at peak to early retrograde conditions. As a result, $P-T$ information is generally only available over a small portion of the retrograde $P-T$ path (e.g., Bohlen, 1987). In this study, $P-T$ information for the northern Wind River Range, an Archean granulite-facies terrane, was obtained using quantitative geothermometry and geobarometry. Variations in $P$ and $T$ during the course of the metamorphism were constrained with mineral assemblages applicable to thermobarometric methods both in the groundmass and in isolated mineral inclusions in garnet porphyroblasts. The Wind River Range, located in western Wyoming (Fig. 1), represents one of the largest exposed metamorphic core complexes in the western United States, comprising the western part of the Archean Wyoming Province (Condie, 1976). The $P-T$ conditions of this terrane add to the available information for the early Archean evolution of the western North American craton.

The Wind River Range consists of a doubly plunging northwest-trending anticline (Branson \& Branson, 1941; Berg, 1962; Smithson et al., 1980) with an exposed Archean core

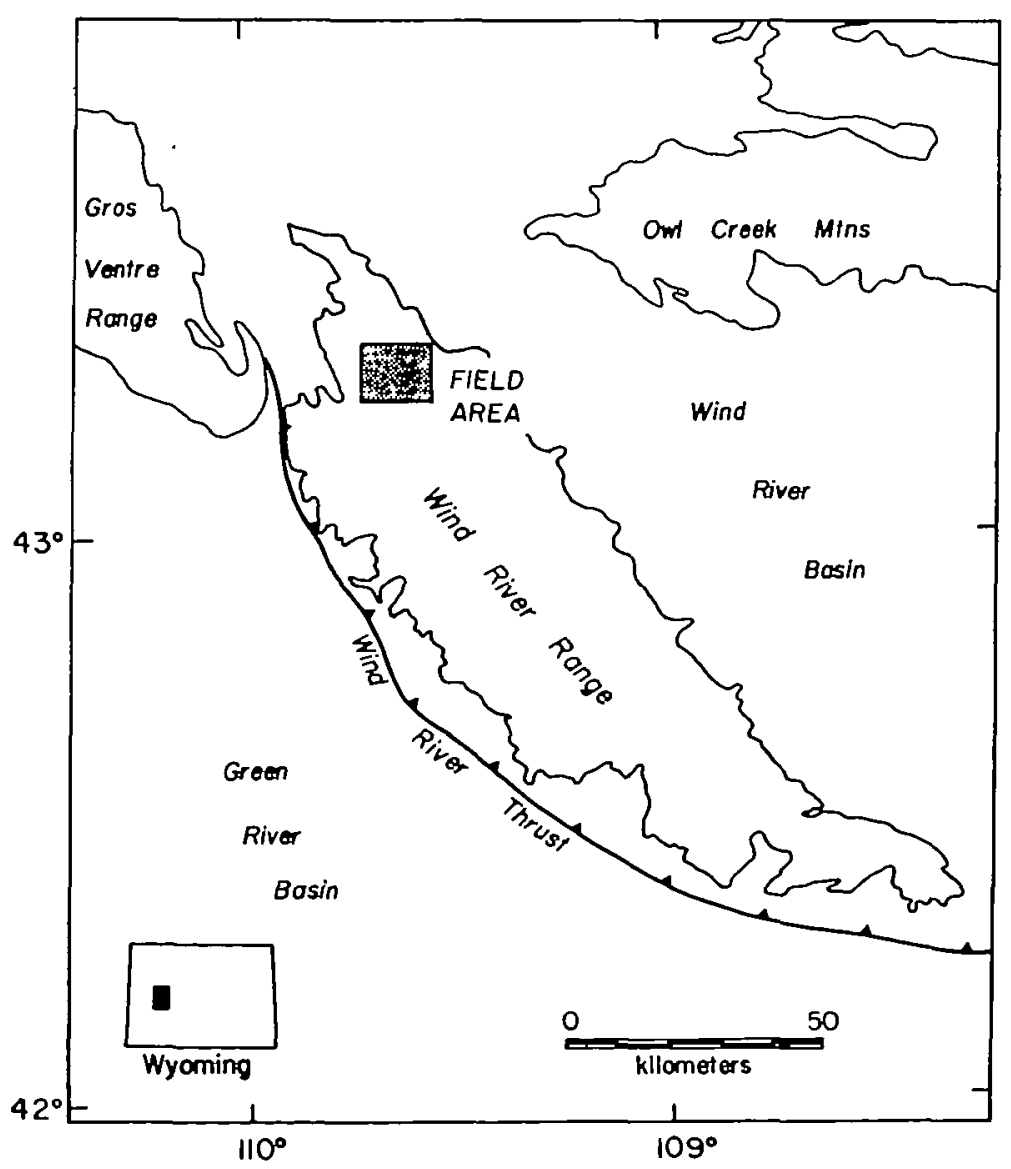

Fig. 1. Map of the Wind River Range. The outline delineates the Archean core complex. The field area in this study is shaded. [See Worl et al. (1984) for greater detail.] 
$\sim 220 \mathrm{~km}$ long and $70 \mathrm{~km}$ wide. This core consists predominantly of granitic orthogneisses and migmatites with blocks of quartzites, metapelites, banded iron formations, amphibolites, and ultramafites (Worl et al., 1984). Age estimates from conventional zircon U-Pb techniques and the SHRIMP ion probe on single zircons range from 3200 to $2600 \mathrm{Ma}$ for pyroxene-bearing granodioritic gneisses (Aleinikoff et al., 1989). The orthogneisses are intruded by granitic plutons dated at $2700 \mathrm{Ma}$ (Bassett \& Giletti, 1963; Naylor et al., 1970), 2630 , and $2550 \mathrm{Ma}$ (Stuckless et al., 1986). Large, unmetamorphosed mafic dikes crosscut all other lithologies and have been dated at 1300-1900 Ma using K-Ar methods for whole-rock samples (Condie et al., 1969) and pyroxene separates (Spall, 1971). The Precambrian core was exposed during the Laramide orogeny along the Wind River fault, which parallels the southwest margin of the range (Berg \& Romberg, 1966; Smithson et al., 1980) (Fig. 1).

The topography of the Wind River Range is extremely variable, ranging from gently rolling hills in the extreme south to high-elevation, deeply glaciated mountains in the north. As a result, geologic investigations have been more extensive in the south (e.g., Bayley, 1963, 1965a, 1965b; Hodge \& Worl, 1965; Pearson et al., 1971; Smithson \& Ebens, 1971; Bayley et al., 1973; Mitra \& Frost, 1981; Cheang et al., 1985). In the central and northern part of the Wind River Range, most work on the Precambrian portions has been at the reconnaissance level (Baker, 1946; Oftendahl, 1953; Granger et al., 1971; Worl et al., 1984). Investigations of the metamorphic conditions of the northern Wind River Range are limited (Perry, 1965; Sharp \& Essene, 1984; Sykes, 1985; Koesterer et al., 1987).

\section{LITHOLOGIC UNITS IN THE NORTHERN WIND RIVER RANGE}

The majority of mapping and sampling in this study was done in the area of the Downs Mountain and Green River Lakes Quadrangles (U.S. Geological Survey 7.5 minute series topographic maps). Felsic orthogneisses and migmatites comprise by far the majority of exposed outcrop, but are not very useful for constraining pressures and temperatures of metamorphism. The more informative 'supracrustal' iron formations, metapelites, and amphibolites are very rare. The locations of these 'supracrustal' units in the present study area have been given by Sharp (1988). Special attention was given to a large banded iron formation (Worl, 1968) located at Rocking Horse Lake (Gannett Peak Quadrangle U.S. Geological Survey Map, 1:100000 series, 1978). Mapping was carried out in the summers of 1982-1985 by Sharp. Essene, M. A. Cosca and G. R. Helffrich all participated in the fieldwork. Mineralogy of representative samples in this study is given in Table 1.

\section{Felsic orthogneisses and migmatites}

The felsic gneisses range from granitic to tonalitic (Worl et al., 1984) and consist of quartz, plagioclase, potassium feldspar, biotite, minor hornblende, and minor orthopyroxene or cummingtonite. Mafic gneisses are generally preserved as stringers within the felsic gneisses, and consist primarily of plagioclase and hornblende, with lesser amounts of biotite, quartz, and rarely clinopyroxene and orthopyroxene. Large, angular blocks of mafic material are also found in the orthogneisses.

\section{Amphibolites}

Amphibolite blocks and boudins are commonly found in association with banded iron formations, metapelites, quartzites, and ultramafites. In the southern Wind River Range, amphibolites and biotite schists are found interbedded with metapelites and iron formations 
TABLE 1

Mineral assemblages for samples used in this study

\begin{tabular}{|c|c|c|c|c|c|c|c|c|c|c|c|c|c|c|c|}
\hline ID no. & $Q z$ & $P g$ & $K s p$ & $G t$ & $O p x$ & $C p x$ & ol & $H n b$ & $B t$ & $M t$ & $I / m$ & $C r d$ & $S i$ & $S p$ & $R u$ \\
\hline \multicolumn{16}{|c|}{ Banded iron formations } \\
\hline A 16 & $x$ & & & & $x$ & $X$ & & $\mathrm{x}$ & & $\mathbf{x}$ & & & & & \\
\hline A22 & $\mathrm{x}$ & $\mathrm{x}$ & & $\mathrm{X}$ & $x$ & $x$ & & & & $\mathrm{X}$ & & & & & \\
\hline W24 & $\mathrm{x}$ & & & $\mathrm{X}$ & $\mathbf{X}$ & & & & & $\mathrm{x}$ & & & & & \\
\hline W57 & $\mathrm{x}$ & & & $\mathbf{X}$ & $X$ & $\mathrm{X}$ & $x$ & & & $\mathrm{x}$ & & & & & \\
\hline W58 & $x$ & $x$ & & $X$ & $\mathbf{X}$ & & & & $\mathrm{X}$ & $\mathrm{x}$ & & & & & \\
\hline W62 & $\mathrm{x}$ & & & $\mathbf{X}$ & $\mathbf{X}$ & $\mathbf{X}$ & & & $\mathrm{X}$ & $\mathrm{x}$ & & & & & \\
\hline T2-E & $\mathrm{x}$ & $\mathrm{x}$ & & $x$ & $X$ & & & $\mathrm{x}$ & & $\mathrm{x}$ & & & & & \\
\hline$T 2-4$ & $\mathrm{x}$ & $\mathrm{x}$ & $\mathrm{X}$ & $\mathbf{X}$ & $\mathbf{X}$ & & & $\mathrm{X}$ & $\mathrm{X}$ & $\mathrm{X}$ & & & & & \\
\hline $\mathrm{T} 2-6$ & $\mathrm{x}$ & & & $\mathrm{X}$ & $\mathbf{X}$ & & & $\mathrm{x}$ & & $\mathrm{x}$ & . & & & & \\
\hline $\mathrm{T} 2-7$ & $\mathrm{x}$ & $\mathrm{x}$ & $\mathrm{X}$ & $\mathbf{X}$ & $\mathbf{X}$ & & & $\mathbf{x}$ & & $\mathbf{x}$ & & & & & \\
\hline T2-9 & $x$ & & & & $\mathbf{X}$ & $\mathrm{X}$ & $\mathrm{X}$ & & & $\mathrm{x}$ & & & & & \\
\hline $\mathrm{T} 2-10$ & $x$ & & & & $X$ & $\mathrm{x}$ & $\mathrm{X}$ & & & $\mathrm{X}$ & & & & & \\
\hline$T 7-6$ & $x$ & $\mathrm{x}$ & $\mathrm{X}$ & $\mathrm{X}$ & $\mathbf{X}$ & & & & $\mathrm{X}$ & $\mathrm{X}$ & & & & & \\
\hline T7-9 & $x$ & & & $X$ & $\mathrm{x}$ & & $\mathrm{x}$ & & & $\mathrm{x}$ & & & & & \\
\hline$T 7-12$ & $x$ & $x$ & & $X$ & $\mathrm{X}$ & & & & $\mathrm{x}$ & $\mathrm{x}$ & & & & & \\
\hline \multicolumn{16}{|c|}{ Metapelites } \\
\hline W78 & $x$ & $\mathrm{x}$ & & $\mathbf{X}$ & & & & & $x$ & & I & $\mathbf{x}$ & I & I & I \\
\hline$W 121$ & $\mathrm{x}$ & $\mathrm{X}$ & $\mathrm{X}$ & $\mathrm{X}$ & & & & & $\mathrm{X}$ & & $\mathbf{X}$ & $\mathrm{X}$ & I & I & I \\
\hline$W 140$ & $\mathrm{x}$ & $x$ & $X$ & $X$ & & & & & $\mathrm{x}$ & & $x$ & $x$ & $X$ & $\mathbf{X}$ & \\
\hline \multicolumn{16}{|c|}{ Amphibolites } \\
\hline$W 120$ & $x$ & $\mathrm{x}$ & & & $X$ & $x$ & & $x$ & & & & & & & \\
\hline$W 123$ & $\mathrm{x}$ & $x$ & & & $X$ & $\mathrm{X}$ & & $\mathrm{x}$ & & & & & & & \\
\hline
\end{tabular}

$\mathrm{X}=$ matrix phase, $\mathrm{I}$ a inclusion in garnet. $\mathrm{Qz}=$ quartz, $\mathrm{Pg}$ a plagıoclase, $\mathrm{Ksp}=$ potassium feldspar, $\mathrm{Gt}=$ garnet, $\mathrm{Opx}=$ orthopyroxene, $\mathrm{Cpx}=$ clinopyroxene, $\mathrm{Ol}=$ olivine, $\mathrm{H}$ nb $=$ hornblende, $\mathrm{Bt}=$ biotite, $\mathrm{Mt}=$ magnetite, $\mathrm{Ilm}=$ ilmenite, $\mathrm{Crd}=$ cordierite, $\mathrm{Si}=$ sillimanite, $\mathrm{Sp}=$ spinel, $\mathrm{Ru}=$ rutile. 
(Bayley, 1963). The amphibolites in the northern Wind River Range consist predominantly of hornblende, quartz, plagioclase, biotite, and potassium feldspar. Minor minerals include garnet, orthopyroxene, clinopyroxene, magnetite, and monazite.

\section{Banded iron formations}

In the northern Wind River Range, banded iron formations are occasionally found as small inclusions within the felsic orthogneiss. One dense cluster of iron formation pods is found in the Downs Mountain Quadrangle surrounding Rocking Horse Lake (Worl, 1968; Sharp, 1988). Sedimentary bedding is well preserved as alternating layers of quartz-rich and pyroxene-magnetite-rich bands, and parallels the elongate direction of the boudins. The layering in the hosting migmatites also parallels the elongate direction of the iron formation lenses. Other smaller outcrops of iron formations are found between Clear Lake (Green River Lakes Quadrangle) and Dads Lake (Downs Mountain Quadrangle) (Sharp, 1988).

The mineralogy of the iron formations consists predominantly of quartz, orthopyroxene, and magnetite, with lesser amounts of garnet, clinopyroxene, olivine, plagioclase, potassium feldspar, hornblende, cummingtonite, and biotite. Accessory minerals include zircon, sphene, and monazite.

\section{Metapelites}

Metapelitic units are found throughout the range (e.g., Worl et al., 1984; Link et al., 1985; Koesterer et al., 1987). In the northern part of the range, thin metapelitic units are associated with the stringers of iron formations between Crescent Lake and Dads Lake (Sharp, 1988). These units consist predominantly of quartz, plagioclase, potassium feldspar, cordierite, biotite, garnet, and sillimanite. Additional minor phases include spinel, ilmenite, and zircon. Rutile, quartz, sillimanite, spinel, cordierite, and plagioclase are occasionally preserved in the cores of garnet and sillimanite porphyroblasts. Corundum is rarely found as inclusions in spinel.

\section{Ultramafites}

Ultramafic bodies are found throughout the Wind River Range (e.g., Worl et al., 1984). The ultramafic bodies in the northern part of the Range either preserve granulite-facies assemblages or are completely hydrated to talc-, serpentine-, chlorite-, amphibole-rich bodies. The anhydrous ultramafic rocks consist of olivine, orthopyroxene, clinoamphibole, chromite, and magnetite, and preserve a granular, coarse-grained fabric.

\section{Quartzites}

Quartzites are well documented in the southern part of the Range (Bayley, 1963). In the present field area, only minor quartzite has been identified in outcrop.

\section{METAMORPHIC CONDITIONS FOR THE NORTHERN WIND RIVER RANGE}

Various geothermometers and geobarometers were used to determine conditions of metamorphism. The highest pressures and temperatures are recorded by mineral inclusions within large garnet porphyroblasts. Mineral chemistries corresponding to lower pressure-temperature conditions are preserved in the groundmass, or matrix mineral 
phases, which can be further divided into core and rim compositions. All mineral compositions were determined with a Cameca CAMEBAX electron microprobe at the University of Michigan with natural and synthetic minerals as standards and operating conditions of $15 \mathrm{kV}$ accelerating voltage and $10 \mu \mathrm{A}$ sample current. ZAF corrections were made with Cameca (PAP) software. Mineral compositions used for thermobarometric determinations in this study are presented in Tables 2-7.

\section{Activity models}

The following activity models were used to calculate pressure-temperature shifts from endmember equilibria for the appropriate geothermometers and geobarometers:

(1) activities of almandine $\left(\mathrm{FeAl}_{2 / 3} \mathrm{SiO}_{4}\right)$ and grossular $\left(\mathrm{CaAl}_{2 / 3} \mathrm{SiO}_{4}\right)$ in garnet were calculated using the mixing model of Ganguly \& Saxena (1984) and the revised mixing parameters of Anovitz \& Essene (1987);

(2) anorthite activities $\left(\mathrm{CaAl}_{2} \mathrm{Si}_{2} \mathrm{O}_{8}\right)$ in plagioclase were calculated following the procedure of Newton et al. (1980);

(3) ferrosilite activities $\left(\mathrm{Fe}_{2} \mathrm{Si}_{2} \mathrm{O}_{6}\right)$ in orthopyroxene were calculated using the procedure of Davidson \& Lindsley (1985);

(4) hercynite activities $\left(\mathrm{FeAl}_{2} \mathrm{O}_{4}\right)$.in spinel were calculated assuming ideal mixing on two sites; and

(5) fayalite activities $\left(\mathrm{Fe}_{2} \mathrm{SiO}_{4}\right)$ in olivine were calculated assuming ideal mixing on two sites.

Pressures and temperatures from garnet porphyroblast inclusions

Minerals enclosed in garnets during porphyroblastic growth become isolated from other phases and may retain their entrapment orientation and/or bulk chemistry (e.g., Rosenfeld, 1970; Tracy, 1982). Therefore, these inclusions may potentially be used to constrain metamorphic conditions at the time of their entrapment (e.g., Thompson et al., 1977) if it can be demonstrated that the inclusions were in equilibrium at the time of entrapment and no post-entrapment volume diffusion or solid-solid reactions occurred. In high-grade rocks, inclusions trapped in garnet porphyroblasts at near-peak metamorphic conditions may become chemically isolated, and record $P-T$ information that may otherwise be lost during cooling by the process of retrograde re-equilibration. Garnet porphyroblasts in the northern Wind River Range are up to $10 \mathrm{~cm}$ in diameter and host a wide variety of minerals, including quartz, plagioclase, biotite, cordierite, spinel, sillimanite, ilmenite, and rutile. Spinel, ilmenite, and rutile are commonly found within the garnet porphyroblasts, but rarely as groundmass phases. Presumably these minerals were consumed during retrogression outside of the garnets, but were isolated from further reaction inside the garnets.

Mineral inclusions in the metapelitic garnets were used to estimate pressures and temperatures during the growth of the garnet. Two equilibria are available from these inclusions with different $\mathrm{d} P / \mathrm{d} T$ slopes. These are

(1) hercynite-quartz-garnet-sillimanite and

(2) garnet-rutile-ilmenite-sillimanite-quartz.

The $P-T$ intersection of these univariant equilibria corrected for solid-solution was assumed to represent peak to near-peak metamorphic conditions of the northern Wind River Range.

Hercynite-quartz-garnet-sillimanite equilibria. The reaction

$$
\begin{aligned}
& 3 \mathrm{FeAl}_{2} \mathrm{O}_{4}+5 \mathrm{SiO}_{2}=\mathrm{Fe}_{3} \mathrm{Al}_{2} \mathrm{Si}_{3} \mathrm{O}_{12}+2 \mathrm{Al}_{2} \mathrm{SiO}_{5} \\
& \text { hercynite quartz almandine sillimanite }
\end{aligned}
$$


TABle 2

Representátive oxide and olivine analyses

\begin{tabular}{|c|c|c|c|c|c|c|c|c|c|c|c|c|}
\hline & $\begin{array}{c}W 78-2 \\
s p\end{array}$ & $\begin{array}{c}W 78-2 \\
s p\end{array}$ & $\begin{array}{c}W 78-2 \\
s p\end{array}$ & $\begin{array}{c}W I 2 l-l \\
s p\end{array}$ & $\begin{array}{c}W I 2 l-2 \\
s p\end{array}$ & $\begin{array}{c}W I 2 l-4 \\
s p\end{array}$ & $\begin{array}{c}W I 2 l-4 \\
s p\end{array}$ & $\begin{array}{c}T 2-9-1 \\
\text { ol }\end{array}$ & $\begin{array}{c}T 2-10 \\
\text { ol }\end{array}$ & $\begin{array}{c}T 7-9-3 \\
\text { ol }\end{array}$ & $\begin{array}{c}W 78-2 \\
i l m\end{array}$ & $\begin{array}{c}W 121-I \\
\text { ilm }\end{array}$ \\
\hline $\begin{array}{l}\mathrm{SiO}_{2} \\
\mathrm{TiO}_{2} \\
\mathrm{Al}_{2} \mathrm{O}_{3} \\
\mathrm{Cr}_{2} \mathrm{O}_{3} \\
\mathrm{Fe}_{2} \mathrm{O}_{3} \\
\mathrm{FeO} \\
\mathrm{MnO} \\
\mathrm{MgO} \\
\mathrm{CaO} \\
\mathrm{ZnO} \\
\mathrm{Sum}\end{array}$ & $\begin{array}{r}0-01 \\
0-01 \\
58.75 \\
1.73 \\
2.41 \\
22.54 \\
0-03 \\
8.79 \\
0-00 \\
5.94 \\
100-21\end{array}$ & $\begin{array}{r}0-01 \\
0-00 \\
58.99 \\
1.83 \\
2.42 \\
22.20 \\
0-00 \\
8.70 \\
0-00 \\
6.44 \\
100-59\end{array}$ & $\begin{array}{r}0-01 \\
0-01 \\
59.37 \\
1.79 \\
2.92 \\
22.78 \\
0.03 \\
9.07 \\
0-01 \\
5.32 \\
101.31\end{array}$ & $\begin{array}{r}0-01 \\
0-09 \\
60-59 \\
1.26 \\
0-00 \\
22.65 \\
0-01 \\
8.60 \\
0-00 \\
6.30 \\
99.51\end{array}$ & $\begin{array}{r}0-00 \\
0-01 \\
59.33 \\
1.93 \\
0.96 \\
23.27 \\
0.01 \\
7.65 \\
0-00 \\
6.97 \\
100.13\end{array}$ & $\begin{array}{r}0-02 \\
0-02 \\
58 \cdot 42 \\
3 \cdot 24 \\
0-47 \\
24.01 \\
0-01 \\
7 \cdot 15 \\
0-01 \\
7.02 \\
100-38\end{array}$ & $\begin{array}{r}0-00 \\
0-02 \\
60-49 \\
1 \cdot 15 \\
0-48 \\
23.92 \\
0.04 \\
8.09 \\
0-03 \\
5.63 \\
99.85\end{array}$ & $\begin{array}{r}30-65 \\
0-00 \\
0-00 \\
0-00 \\
0-00 \\
67 \cdot 92 \\
0-61 \\
2 \cdot 14 \\
0-06 \\
\text { n.d. } \\
101 \cdot 37\end{array}$ & $\begin{array}{r}30-67 \\
0-00 \\
0-00 \\
0-02 \\
0-00 \\
67 \cdot 71 \\
0.45 \\
1.91 \\
0-03 \\
\text { n.d. } \\
100-79\end{array}$ & $\begin{array}{r}30-99 \\
0-00 \\
0.00 \\
0-00 \\
0-00 \\
67 \cdot 17 \\
0.71 \\
2 \cdot 15 \\
0-05 \\
\text { n.d. } \\
101.06\end{array}$ & $\begin{array}{r}0-01 \\
51 \cdot 29 \\
0-00 \\
0-09 \\
2.42 \\
43.85 \\
0-23 \\
1 \cdot 14 \\
0-01 \\
0-03 \\
99.07\end{array}$ & $\begin{array}{r}0-03 \\
52.23 \\
0-00 \\
0-14 \\
0-91 \\
43.78 \\
0-13 \\
1.68 \\
0-02 \\
0-09 \\
99.01\end{array}$ \\
\hline $\begin{array}{l}\mathrm{Si} \\
\mathrm{Ti} \\
\mathrm{Al} \\
\mathrm{Cr} \\
\mathrm{Fe}^{3+} \\
\mathrm{Fe}^{2+} \\
\mathrm{Mn} \\
\mathrm{Mg} \\
\mathrm{Ca} \\
\mathrm{Zn}\end{array}$ & $\begin{array}{l}0-00 \\
0-00 \\
1.91 \\
0-04 \\
0-05 \\
0-52 \\
0-00 \\
0-36 \\
0-00 \\
0-12\end{array}$ & $\begin{array}{l}0-00 \\
0-00 \\
1.91 \\
0-04 \\
0-05 \\
0-51 \\
0-00 \\
0.36 \\
0-00 \\
0.13\end{array}$ & $\begin{array}{l}0-00 \\
0-00 \\
1.91 \\
0-04 \\
0-06 \\
0.52 \\
0-00 \\
0.37 \\
0-00 \\
0-11\end{array}$ & $\begin{array}{l}0-00 \\
0-00 \\
1.96 \\
0-03 \\
0-00 \\
0-52 \\
0-00 \\
0-35 \\
0-00 \\
0-13\end{array}$ & $\begin{array}{l}0-00 \\
0-00 \\
1.94 \\
0-04 \\
0-02 \\
0-54 \\
0-00 \\
0-32 \\
0-00 \\
0-14\end{array}$ & $\begin{array}{l}0-00 \\
0-00 \\
1.92 \\
0-07 \\
0-01 \\
0-56 \\
0-00 \\
0-33 \\
0-00 \\
0-14\end{array}$ & $\begin{array}{l}0-00 \\
0-00 \\
1.96 \\
0-03 \\
0-01 \\
0-55 \\
0-00 \\
0-33 \\
0-00 \\
0-11\end{array}$ & $\begin{array}{c}1.01 \\
0-00 \\
0-00 \\
0-00 \\
0-00 \\
1.87 \\
0-02 \\
0-10 \\
0-00 \\
\text { n.d. }\end{array}$ & $\begin{array}{c}1-02 \\
0-00 \\
0-00 \\
0-00 \\
0-00 \\
1.88 \\
0=01 \\
0-09 \\
0-00 \\
\text { n.d. }\end{array}$ & $\begin{array}{c}1.02 \\
0-00 \\
0-00 \\
0-00 \\
0-00 \\
1.85 \\
0-02 \\
0-11 \\
0-00 \\
\text { n.d. }\end{array}$ & $\begin{array}{l}0-00 \\
0-98 \\
0-00 \\
0-00 \\
0-05 \\
0-93 \\
0-00 \\
0-04 \\
0-00 \\
0-00\end{array}$ & $\begin{array}{l}0-00 \\
0-99 \\
0-00 \\
0-00 \\
0-02 \\
0-92 \\
0-00 \\
0-06 \\
0-00 \\
0-00\end{array}$ \\
\hline \multirow[t]{2}{*}{ Activity } & $0-50$ & 0.49 & 0.50 & 0.51 & $0-52$ & $0-54$ & $0-54$ & 0.94 & 0.95 & 0.94 & $0-91$ & $0-91$ \\
\hline & $\begin{array}{c}T 7-6 \\
m t\end{array}$ & $\begin{array}{c}T 7-9 \\
m t\end{array}$ & $\begin{array}{c}T 7-12 \\
m t\end{array}$ & & & & & & & & & \\
\hline $\begin{array}{l}\mathrm{TiO}_{2} \\
\mathrm{Al}_{2} \mathrm{O}_{3} \\
\mathrm{Fe}_{2} \mathrm{O}_{3} \\
\mathrm{FcO} \\
\mathrm{Sum}\end{array}$ & $\begin{array}{r}3.95 \\
1.49 \\
59.12 \\
34.75 \\
99.31\end{array}$ & $\begin{array}{r}2.23 \\
0.64 \\
63.52 \\
33.04 \\
99.42\end{array}$ & $\begin{array}{r}1.63 \\
1.07 \\
64.45 \\
32.68 \\
99.83\end{array}$ & & & & & & & & & \\
\hline $\begin{array}{l}\mathrm{Ti} \\
\mathrm{Al} \\
\mathrm{Fe}^{3+} \\
\mathrm{Fe}^{2+}\end{array}$ & $\begin{array}{l}0.11 \\
0-07 \\
1 \cdot 71 \\
1 \cdot 11\end{array}$ & $\begin{array}{l}0-07 \\
0-03 \\
1.84 \\
1.07\end{array}$ & $\begin{array}{l}0-05 \\
0-05 \\
1.86 \\
1.05\end{array}$ & & & & & & & & & \\
\hline$X(\mathrm{mt})$ & $0-85$ & $0-92$ & $0-93$ & & & & & & & & & \\
\hline
\end{tabular}

Normalization: sp, ol, $\mathrm{mt}$-three cations; il-two cations. Abbreviations as in Table 1. $a$ (fayalite) $=a\left(\mathrm{Fe}_{2} \mathrm{SiO}_{4}\right) ; a($ hercynite $)=a\left(\mathrm{FeAl}{ }_{2} \mathrm{O}_{4}\right) ; a($ ilmenite $)$ $=a\left(\mathrm{FeTiO}_{3}\right)$ 
TABLE 3

Representative garnet analyses

\begin{tabular}{|c|c|c|c|c|c|c|c|c|c|c|}
\hline & $\begin{array}{c}\text { A22-2 } \\
\text { core }\end{array}$ & $\underset{\text { rim }}{A 22-2}$ & $\begin{array}{l}\text { W24-I } \\
\text { core }\end{array}$ & $\begin{array}{c}\text { W24-I } \\
\text { rim }\end{array}$ & $\begin{array}{c}\text { W57-3 } \\
\text { core }\end{array}$ & $\begin{array}{c}\text { W57-3 } \\
\text { rim }\end{array}$ & $\begin{array}{c}\text { W58-I } \\
\text { core }\end{array}$ & $\underset{\text { rim }}{W 58-I}$ & $\begin{array}{c}\text { W58-4 } \\
\text { core }\end{array}$ & $\underset{\text { Wim }}{\text { W58-4 }}$ \\
\hline $\begin{array}{l}\mathrm{SiO}_{2} \\
\mathrm{TiO}_{2} \\
\mathrm{Al}_{2} \mathrm{O}_{3} \\
\mathrm{Cr}_{2} \mathrm{O}_{3} \\
\mathrm{Fe}_{2} \mathrm{O}_{3} \\
\mathrm{FeO} \\
\mathrm{MnO} \\
\mathrm{MgO} \\
\mathrm{CaO} \\
\mathrm{Sum}\end{array}$ & $\begin{array}{r}37.55 \\
0.00 \\
19.59 \\
0-02 \\
1.62 \\
30.24 \\
1.06 \\
0.56 \\
8.83 \\
99.47\end{array}$ & $\begin{array}{r}37.25 \\
0-00 \\
19.65 \\
0-00 \\
1.45 \\
30-06 \\
0.76 \\
0-60 \\
9.08 \\
98.85\end{array}$ & $\begin{array}{r}37.46 \\
0-03 \\
20.51 \\
0-04 \\
0.49 \\
36.35 \\
0-09 \\
2.38 \\
2.53 \\
99.87\end{array}$ & $\begin{array}{r}37.14 \\
0-02 \\
20.51 \\
0.03 \\
0-65 \\
36.53 \\
0.16 \\
2.03 \\
2.94 \\
100-00\end{array}$ & $\begin{array}{r}36.76 \\
0-05 \\
19.97 \\
0-05 \\
1.47 \\
31.08 \\
0-42 \\
0.86 \\
8.64 \\
99.30\end{array}$ & $\begin{array}{r}37 \cdot 10 \\
0-03 \\
20.32 \\
0-05 \\
0.99 \\
32.09 \\
0-54 \\
0-57 \\
8.35 \\
100-03\end{array}$ & $\begin{array}{r}37.79 \\
0-00 \\
20-42 \\
0-05 \\
0-49 \\
34.48 \\
0-47 \\
1.43 \\
4.94 \\
100-06\end{array}$ & $\begin{array}{r}37.47 \\
0.03 \\
20.29 \\
0-01 \\
0.98 \\
35.33 \\
0.38 \\
1.03 \\
5.11 \\
100-63\end{array}$ & $\begin{array}{r}36.91 \\
0.00 \\
21.02 \\
0-06 \\
0.67 \\
35.22 \\
0.45 \\
1.51 \\
5.35 \\
101.19\end{array}$ & $\begin{array}{r}36.82 \\
0-01 \\
20-55 \\
0-01 \\
0-82 \\
34.37 \\
0-44 \\
1.00 \\
6 \cdot 10 \\
100-11\end{array}$ \\
\hline $\begin{array}{l}\mathrm{Si} \\
\mathrm{Ti} \\
\mathrm{Al} \\
\mathrm{Cr} \\
\mathrm{Fe}^{3+} \\
\mathrm{Fe}^{2+} \\
\mathrm{Mn} \\
\mathrm{Mg} \\
\mathrm{Ca}\end{array}$ & $\begin{array}{l}3-09 \\
0-00 \\
1.90 \\
0-00 \\
0-10 \\
2-08 \\
0-07 \\
0-07 \\
0.78\end{array}$ & $\begin{array}{l}3-07 \\
0-00 \\
1.91 \\
0-00 \\
0-09 \\
2-07 \\
0-05 \\
0-07 \\
0-80\end{array}$ & $\begin{array}{l}3.06 \\
0-00 \\
1.97 \\
0-00 \\
0-03 \\
2.48 \\
0-01 \\
0-29 \\
0-22\end{array}$ & $\begin{array}{l}3.02 \\
0-00 \\
1.96 \\
0-00 \\
0-04 \\
2.48 \\
0.01 \\
0.25 \\
0.26\end{array}$ & $\begin{array}{l}2.99 \\
0-00 \\
1.91 \\
0-00 \\
0-09 \\
2.11 \\
0-03 \\
0-10 \\
0.75\end{array}$ & $\begin{array}{l}3-00 \\
0-00 \\
1.94 \\
0-00 \\
0-06 \\
2.17 \\
0-04 \\
0-07 \\
0-72\end{array}$ & $\begin{array}{l}3-09 \\
0-00 \\
1.97 \\
0-00 \\
0-03 \\
2.36 \\
0-03 \\
0-18 \\
0-43\end{array}$ & $\begin{array}{l}3-04 \\
0-00 \\
1.94 \\
0-00 \\
0-06 \\
2.40 \\
0-03 \\
0-12 \\
0-44\end{array}$ & $\begin{array}{l}2.92 \\
0-00 \\
1.96 \\
0-00 \\
0-04 \\
2.33 \\
0-03 \\
0-18 \\
0-45\end{array}$ & $\begin{array}{l}2.97 \\
0-00 \\
1.95 \\
0-00 \\
0-05 \\
2.32 \\
0-03 \\
0.12 \\
0.53\end{array}$ \\
\hline $\begin{array}{l}X(\mathrm{Alm}) \\
X(\mathrm{Py}) \\
X(\mathrm{Gr}) \\
a(\mathrm{Alm}) \\
a(\mathrm{Gr})\end{array}$ & $\begin{array}{l}0-693 \\
0-023 \\
0-260 \\
0-619 \\
0-324\end{array}$ & $\begin{array}{l}0-692 \\
0-023 \\
0-268 \\
0-621 \\
0-331\end{array}$ & $\begin{array}{l}0-827 \\
0-097 \\
0-073 \\
0-824 \\
0-063\end{array}$ & $\begin{array}{l}0-827 \\
0-083 \\
0-087 \\
0-817 \\
0-077\end{array}$ & $\begin{array}{l}0-706 \\
0-033 \\
0.251 \\
0.638 \\
0.299\end{array}$ & $\begin{array}{l}0.723 \\
0-023 \\
0-240 \\
0-654 \\
0-301\end{array}$ & $\begin{array}{l}0-787 \\
0-060 \\
0-143 \\
0-753 \\
0-141\end{array}$ & $\begin{array}{l}0-803 \\
0-040 \\
0-147 \\
0-761 \\
0-154\end{array}$ & $\begin{array}{l}0.779 \\
0-060 \\
0.151 \\
0.743 \\
0-152\end{array}$ & $\begin{array}{l}0.773 \\
0-040 \\
0.177 \\
0.725 \\
0.194\end{array}$ \\
\hline
\end{tabular}


TABLE 3 (Continued)

\begin{tabular}{|c|c|c|c|c|c|c|c|c|c|c|}
\hline & $\begin{array}{c}\text { W62-4 } \\
\text { core }\end{array}$ & $\begin{array}{c}\text { W62-4 } \\
\mathrm{rim}\end{array}$ & $\begin{array}{l}\text { W78-2 } \\
\text { core }\end{array}$ & $\begin{array}{c}\text { WI2I-I } \\
\text { core }\end{array}$ & $\begin{array}{c}W 12 I-2 \\
\text { core }\end{array}$ & $\begin{array}{l}\text { WI2I-4 } \\
\text { core }\end{array}$ & $\begin{array}{c}\text { WI40-1 } \\
\text { core }\end{array}$ & $\begin{array}{c}W I 40-1 \\
\mathrm{rim}\end{array}$ & $\begin{array}{c}W 140-2 \\
\text { core }\end{array}$ & $\begin{array}{l}\text { W140-2 } \\
\text { rim }\end{array}$ \\
\hline $\begin{array}{l}\mathrm{SiO}_{2} \\
\mathrm{TiO}_{2} \\
\mathrm{Al}_{2} \mathrm{O}_{3} \\
\mathrm{Cr}_{2} \mathrm{O}_{3} \\
\mathrm{Fe}_{2} \mathrm{O}_{3} \\
\mathrm{FeO} \\
\mathrm{MnO} \\
\mathrm{MgO} \\
\mathrm{CaO} \\
\mathrm{Sum}\end{array}$ & $\begin{array}{r}37 \cdot 43 \\
0-00 \\
20-80 \\
0-01 \\
0-67 \\
35 \cdot 37 \\
0-21 \\
3 \cdot 70 \\
2 \cdot 17 \\
100-36\end{array}$ & $\begin{array}{r}37.40 \\
0-01 \\
20.38 \\
0.03 \\
0.49 \\
35.65 \\
0.18 \\
2.74 \\
2.26 \\
99.13\end{array}$ & $\begin{array}{r}38 \cdot 78 \\
0-01 \\
21 \cdot 54 \\
0-09 \\
0-00 \\
31 \cdot 50 \\
0-83 \\
7 \cdot 39 \\
0.99 \\
101 \cdot 13\end{array}$ & $\begin{array}{r}38 \cdot 76 \\
0-01 \\
22 \cdot 42 \\
0-13 \\
0-00 \\
28 \cdot 73 \\
0-51 \\
8 \cdot 77 \\
1 \cdot 13 \\
100-46\end{array}$ & $\begin{array}{r}38 \cdot 67 \\
0-01 \\
22 \cdot 20 \\
0-08 \\
0-00 \\
30-21 \\
0-49 \\
7 \cdot 73 \\
1 \cdot 25 \\
100-63\end{array}$ & $\begin{array}{r}38 \cdot 24 \\
0-00 \\
21 \cdot 98 \\
0-06 \\
0-03 \\
32 \cdot 55 \\
0-61 \\
6 \cdot 46 \\
0-95 \\
100-88\end{array}$ & $\begin{array}{r}37 \cdot 85 \\
0-04 \\
22 \cdot 12 \\
0-05 \\
0-00 \\
35 \cdot 21 \\
0-62 \\
4.55 \\
1 \cdot 16 \\
101 \cdot 60\end{array}$ & $\begin{array}{r}36 \cdot 31 \\
0-02 \\
21 \cdot 39 \\
0-04 \\
0-00 \\
36.15 \\
0-71 \\
3.32 \\
1.48 \\
99.41\end{array}$ & $\begin{array}{r}38 \cdot 29 \\
0-00 \\
21 \cdot 85 \\
0-01 \\
0-00 \\
35-35 \\
0-66 \\
4 \cdot 70 \\
1.01 \\
101 \cdot 88\end{array}$ & $\begin{array}{r}36.36 \\
0-05 \\
20-99 \\
0-03 \\
0-00 \\
36.77 \\
0.71 \\
2.74 \\
1.55 \\
99.46\end{array}$ \\
\hline $\begin{array}{l}\mathrm{Si} \\
\mathrm{Ti} \\
\mathrm{Al} \\
\mathrm{Cr} \\
\mathrm{Fe}^{3+} \\
\mathrm{Fe}^{2+} \\
\mathrm{Mn} \\
\mathrm{Mg} \\
\mathrm{Ca}\end{array}$ & $\begin{array}{l}2.99 \\
0-00 \\
1.96 \\
0-00 \\
0-04 \\
2.36 \\
0.01 \\
0.44 \\
0.19\end{array}$ & $\begin{array}{l}3-07 \\
0-00 \\
1.97 \\
0-00 \\
0-03 \\
2.45 \\
0-01 \\
0.34 \\
0-20\end{array}$ & $\begin{array}{l}3.00 \\
0-00 \\
1.97 \\
0-01 \\
0-02 \\
2.01 \\
0-05 \\
0-85 \\
0-08\end{array}$ & $\begin{array}{l}2.98 \\
0-00 \\
2.03 \\
0-01 \\
0-00 \\
1.85 \\
0-03 \\
1.00 \\
0-09\end{array}$ & $\begin{array}{l}2.99 \\
0-00 \\
2.02 \\
0-00 \\
0-00 \\
1.95 \\
0-03 \\
0.89 \\
0.10\end{array}$ & $\begin{array}{l}2 \cdot 98 \\
0-00 \\
2 \cdot 02 \\
0-00 \\
0-00 \\
2 \cdot 12 \\
0-04 \\
0-75 \\
0-08\end{array}$ & $\begin{array}{l}2.97 \\
0-00 \\
2-05 \\
0-00 \\
0-00 \\
2.31 \\
0-04 \\
0-53 \\
0.10\end{array}$ & $\begin{array}{l}2.94 \\
0-00 \\
2.04 \\
0-00 \\
0-00 \\
2.44 \\
0-05 \\
0-40 \\
0.13\end{array}$ & $\begin{array}{l}3-00 \\
0-00 \\
2-01 \\
0-00 \\
0-00 \\
2-31 \\
0-04 \\
0-55 \\
0-08\end{array}$ & $\begin{array}{l}2.97 \\
0-00 \\
2-01 \\
0-00 \\
0-00 \\
2.50 \\
0-05 \\
0-33 \\
0-13\end{array}$ \\
\hline $\begin{array}{l}X(\mathrm{Alm}) \\
X(\mathrm{Py}) \\
X(\mathrm{Gr}) \\
a(\mathrm{Alm}) \\
a(\mathrm{Gr})\end{array}$ & $\begin{array}{l}0.787 \\
0.147 \\
0-063 \\
0.802 \\
0-057\end{array}$ & $\begin{array}{l}0.817 \\
0-113 \\
0-067 \\
0-821 \\
0-061\end{array}$ & $\begin{array}{l}0.672 \\
0.284 \\
0-027 \\
0.750 \\
0-030\end{array}$ & $\begin{array}{l}0-623 \\
0-337 \\
0-030 \\
0-718 \\
0-040\end{array}$ & $\begin{array}{l}0-657 \\
0-300 \\
0-034 \\
0-737 \\
0-041\end{array}$ & $\begin{array}{l}0-709 \\
0-251 \\
0-027 \\
0-775 \\
0-035\end{array}$ & $\begin{array}{l}0-775 \\
0-178 \\
0-034 \\
0-808 \\
0-040\end{array}$ & $\begin{array}{l}0-808 \\
0-132 \\
0-043 \\
0-824 \\
0-036\end{array}$ & $\begin{array}{l}0.775 \\
0.185 \\
0-027 \\
0.815 \\
0-025\end{array}$ & $\begin{array}{l}0-831 \\
0-110 \\
0-043 \\
0-839 \\
0-034\end{array}$ \\
\hline
\end{tabular}


TABLE 3 (Continued)

\begin{tabular}{|c|c|c|c|c|c|c|c|c|c|c|c|}
\hline & $\begin{array}{c}\text { WI40-5 } \\
\text { core }\end{array}$ & $\begin{array}{l}T 2-E \\
\text { core }\end{array}$ & $\begin{array}{c}T 2-E \\
\text { rim }\end{array}$ & $\begin{array}{c}T 2-4-2 \\
\text { core }\end{array}$ & $\begin{array}{c}T 2-4-2 \\
\text { rim }\end{array}$ & $\begin{array}{c}T 2-6-1 \\
\text { core }\end{array}$ & $\begin{array}{c}\text { T2-6-1 } \\
\text { rim }\end{array}$ & $\begin{array}{c}T 2-7-3 \\
\text { core }\end{array}$ & $\begin{array}{l}\text { T2-7-3 } \\
\text { rim }\end{array}$ & $\begin{array}{l}77-12 \\
\text { core }\end{array}$ & $\underset{\mathrm{rim}}{T 7-12}$ \\
\hline $\begin{array}{l}\mathrm{SiO}_{2} \\
\mathrm{TiO}_{2} \\
\mathrm{Al}_{2} \mathrm{O}_{3} \\
\mathrm{Cr}_{2} \mathrm{O}_{3} \\
\mathrm{Fe}_{2} \mathrm{O}_{3} \\
\mathrm{FeO} \\
\mathrm{MeO} \\
\mathrm{MgO} \\
\mathrm{CaO} \\
\mathrm{Sum}\end{array}$ & $\begin{array}{r}37.92 \\
0-05 \\
22.20 \\
0.05 \\
0.00 \\
35.45 \\
0.70 \\
4.63 \\
1.05 \\
102.05\end{array}$ & $\begin{array}{r}37.47 \\
0-00 \\
20-82 \\
0-06 \\
0-49 \\
35.45 \\
0.53 \\
1.40 \\
4.66 \\
100-88\end{array}$ & $\begin{array}{r}37 \cdot 63 \\
0-00 \\
21 \cdot 14 \\
0-00 \\
0.00 \\
36 \cdot 12 \\
0.47 \\
0.92 \\
5 \cdot 10 \\
101 \cdot 38\end{array}$ & $\begin{array}{r}36.21 \\
0-00 \\
20-42 \\
0-02 \\
0.65 \\
34.92 \\
0.57 \\
1.40 \\
4.71 \\
98.89\end{array}$ & $\begin{array}{r}36.64 \\
0-00 \\
20-77 \\
0-02 \\
0.33 \\
35.53 \\
0.72 \\
1.03 \\
4.88 \\
99.93\end{array}$ & $\begin{array}{r}36.88 \\
0-00 \\
20-39 \\
0-00 \\
0.99 \\
34.25 \\
0.57 \\
1.47 \\
5.48 \\
100-04\end{array}$ & $\begin{array}{r}36 \cdot 91 \\
0-00 \\
20-24 \\
0-05 \\
0-98 \\
33.72 \\
0-56 \\
0-98 \\
6.34 \\
99.78\end{array}$ & $\begin{array}{r}36.94 \\
0.04 \\
20.14 \\
0-00 \\
1.31 \\
35 \cdot 12 \\
0-45 \\
1 \cdot 19 \\
4.99 \\
100-19\end{array}$ & $\begin{array}{r}36.56 \\
0-00 \\
20-48 \\
0-04 \\
0.99 \\
35.44 \\
0-45 \\
1.11 \\
5.31 \\
100-37\end{array}$ & $\begin{array}{r}37.57 \\
0-01 \\
20-88 \\
0-05 \\
0.50 \\
36.15 \\
0.52 \\
1.15 \\
4.62 \\
101.44\end{array}$ & $\begin{array}{r}37.61 \\
0-00 \\
21.01 \\
0-03 \\
0.33 \\
36.31 \\
0.57 \\
1.03 \\
4.67 \\
101.55\end{array}$ \\
\hline $\begin{array}{l}\mathrm{Si} \\
\mathrm{Ti} \\
\mathrm{Al} \\
\mathrm{Cr} \\
\mathrm{Fe}^{3+} \\
\mathrm{Fe}^{2+} \\
\mathrm{Mn} \\
\mathrm{Mg} \\
\mathrm{Ca}\end{array}$ & $\begin{array}{l}2.96 \\
0-00 \\
2.04 \\
0-00 \\
0-00 \\
2.32 \\
0-05 \\
0-54 \\
0-09\end{array}$ & $\begin{array}{l}3-02 \\
0-00 \\
1.97 \\
0-00 \\
0-03 \\
2.39 \\
0-04 \\
0.17 \\
0-40\end{array}$ & $\begin{array}{l}3-02 \\
0-00 \\
2-00 \\
0-00 \\
0-00 \\
2.42 \\
0-03 \\
0.11 \\
0-44\end{array}$ & $\begin{array}{l}2.95 \\
0-00 \\
1.96 \\
0-00 \\
0.04 \\
2.38 \\
0-04 \\
0.17 \\
0.41\end{array}$ & $\begin{array}{l}2.96 \\
0-00 \\
1.98 \\
0-00 \\
0-02 \\
2.40 \\
0-05 \\
0-12 \\
0.42\end{array}$ & $\begin{array}{l}2.98 \\
0-00 \\
1.94 \\
0-00 \\
0-06 \\
2.31 \\
0-04 \\
0.18 \\
0.47\end{array}$ & $\begin{array}{l}3-00 \\
0-00 \\
1.94 \\
0-00 \\
0-06 \\
2.29 \\
0-04 \\
0.12 \\
0-55\end{array}$ & $\begin{array}{l}2.99 \\
0-00 \\
1.92 \\
0-00 \\
0-08 \\
2.38 \\
0-03 \\
0-14 \\
0.43\end{array}$ & $\begin{array}{l}2.94 \\
0-00 \\
1.94 \\
0-00 \\
0-06 \\
2.38 \\
0-03 \\
0.13 \\
0.46\end{array}$ & $\begin{array}{l}3.01 \\
0-00 \\
1.97 \\
0-00 \\
0-03 \\
2.42 \\
0-04 \\
0.14 \\
0.40\end{array}$ & $\begin{array}{l}3-01 \\
0-00 \\
1.98 \\
0-00 \\
0-02 \\
2.43 \\
0-04 \\
0-12 \\
0-40\end{array}$ \\
\hline $\begin{array}{l}X(\mathrm{Alm}) \\
X(\mathrm{Py}) \\
X(\mathrm{Gr}) \\
a(\mathrm{~A} \mid \mathrm{m}) \\
a(\mathrm{Gr})\end{array}$ & $\begin{array}{l}0.773 \\
0.180 \\
0-030 \\
0.811 \\
0-027\end{array}$ & $\begin{array}{l}0.797 \\
0.057 \\
0.133 \\
0.769 \\
0.126\end{array}$ & $\begin{array}{l}0.807 \\
0.037 \\
0.147 \\
0.768 \\
0.149\end{array}$ & $\begin{array}{l}0.793 \\
0.057 \\
0.137 \\
0.761 \\
0.132\end{array}$ & $\begin{array}{l}0-803 \\
0-040 \\
0-140 \\
0-764 \\
0-142\end{array}$ & $\begin{array}{l}0-770 \\
0-060 \\
0-157 \\
0-732 \\
0-160\end{array}$ & $\begin{array}{l}0-763 \\
0-040 \\
0-183 \\
0.712 \\
0-207\end{array}$ & $\begin{array}{l}0-799 \\
0-047 \\
0-144 \\
0-762 \\
0-141\end{array}$ & $\begin{array}{l}0-793 \\
0-043 \\
0-153 \\
0-752 \\
0-159\end{array}$ & $\begin{array}{l}0-807 \\
0-047 \\
0-133 \\
0-781 \\
0-122\end{array}$ & $\begin{array}{l}0-813 \\
0-040 \\
0-134 \\
0-779 \\
0-131\end{array}$ \\
\hline
\end{tabular}

Normalization: five cations, excluding Si. Si total assumed as 3.0 for $\mathrm{Fe}^{3+}$ determination. Core and rim activities at 750 and $650^{\circ} \mathrm{C}$, except for W78 and $\mathrm{W} 121$ at $800^{\circ} \mathrm{C} . a(\mathrm{Alm})=a\left(\mathrm{FeAl}_{2 / 3} \mathrm{SiO}_{4}\right), a(\mathrm{Gr})=a\left(\mathrm{CaAl}_{2 / 3} \mathrm{SiO}_{4}\right), \mathrm{Py}=$ pyrope. 


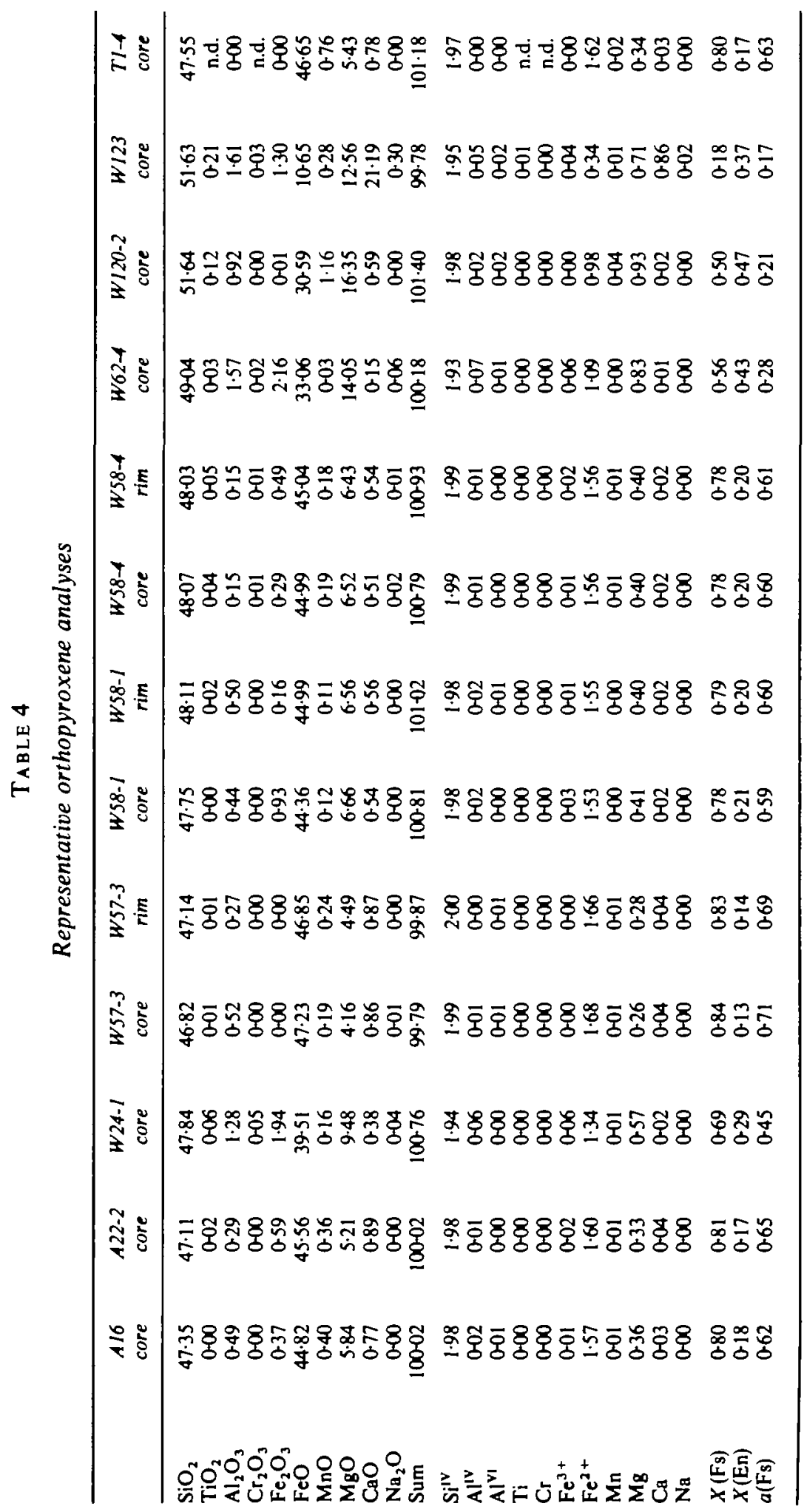


Table 4 (Continued)

\begin{tabular}{|c|c|c|c|c|c|c|c|c|c|c|c|c|c|}
\hline & $\begin{array}{l}T 2-E \\
\text { core }\end{array}$ & $\underset{\mathrm{rim}}{T 2+E}$ & $\begin{array}{l}\text { T2-4-2 } \\
\text { core }\end{array}$ & $\underset{\mathrm{rim}}{T 2-4-2}$ & $\begin{array}{c}T 2-6 \cdot 1 \\
\text { core }\end{array}$ & $\begin{array}{c}\text { T2-6-1 } \\
\text { rim }\end{array}$ & $\begin{array}{c}\text { T2-7-3 } \\
\text { core }\end{array}$ & $\begin{array}{c}T 2-7-3 \\
\text { rim }\end{array}$ & $\begin{array}{l}T 2-9 \\
\text { core }\end{array}$ & $\begin{array}{c}T 2-10 \\
\text { core }\end{array}$ & $\begin{array}{l}T 7-9 \\
\text { core }\end{array}$ & $\begin{array}{l}77-12 \\
\text { core }\end{array}$ & $\begin{array}{c}77-12 \\
\mathrm{rim}\end{array}$ \\
\hline $\begin{array}{l}\mathrm{SiO}_{2} \\
\mathrm{TiO}_{2} \\
\mathrm{Al}_{2} \mathrm{O}_{3} \\
\mathrm{Cr}_{2} \mathrm{O}_{3} \\
\mathrm{Fe}_{2} \mathrm{O}_{3} \\
\mathrm{FeO} \\
\mathrm{MnO} \\
\mathrm{MgO} \mathrm{O} \\
\mathrm{CaO} \\
\mathrm{Na}_{2} \mathrm{O} \\
\mathrm{Sum}\end{array}$ & $\begin{array}{r}47.86 \\
0-01 \\
0.53 \\
0-00 \\
0-00 \\
45-01 \\
0.13 \\
6.36 \\
0-50 \\
0-00 \\
100-40\end{array}$ & $\begin{array}{r}48 \cdot 40 \\
0-00 \\
0-38 \\
0-00 \\
0-00 \\
44 \cdot 75 \\
0.17 \\
6.59 \\
0-49 \\
0-00 \\
100-77\end{array}$ & $\begin{array}{r}47.51 \\
0-00 \\
0-44 \\
0-02 \\
0-20 \\
44 \cdot 18 \\
0-25 \\
6.51 \\
0-58 \\
0-01 \\
99.69\end{array}$ & $\begin{array}{r}48.31 \\
0-00 \\
0.12 \\
0-01 \\
0-00 \\
44.37 \\
0-18 \\
6.51 \\
0-47 \\
0-00 \\
99.97\end{array}$ & $\begin{array}{r}48 \cdot 53 \\
0-04 \\
0-36 \\
0-03 \\
0-06 \\
43 \cdot 69 \\
0-21 \\
7 \cdot 24 \\
0-60 \\
0-00 \\
100-74\end{array}$ & $\begin{array}{r}48.09 \\
0-01 \\
0-32 \\
0-00 \\
1.43 \\
42.97 \\
0-22 \\
7.68 \\
0-49 \\
0-00 \\
101.19\end{array}$ & $\begin{array}{r}48.12 \\
0-06 \\
0.36 \\
0-06 \\
0-00 \\
45.48 \\
0-15 \\
5.96 \\
0-69 \\
0-00 \\
100.88\end{array}$ & $\begin{array}{r}47 \cdot 76 \\
0-05 \\
0-34 \\
0-09 \\
0-51 \\
44 \cdot 70 \\
0-15 \\
6-39 \\
0-68 \\
0-00 \\
100-69\end{array}$ & $\begin{array}{r}47.63 \\
0-00 \\
0-10 \\
0-00 \\
0-00 \\
46.53 \\
0.56 \\
4.84 \\
0-81 \\
0-00 \\
100-47\end{array}$ & $\begin{array}{r}48.39 \\
0-00 \\
0-11 \\
0-00 \\
0-00 \\
46.23 \\
0.43 \\
4.89 \\
0-87 \\
0-01 \\
100.92\end{array}$ & $\begin{array}{r}47.73 \\
0-01 \\
0-31 \\
0-04 \\
0.33 \\
46.35 \\
0.38 \\
5.39 \\
0-44 \\
0-04 \\
10.03\end{array}$ & $\begin{array}{r}48 \cdot 10 \\
0-02 \\
0-53 \\
0-07 \\
0.05 \\
45 \cdot 16 \\
0-25 \\
6.28 \\
0-57 \\
0-04 \\
101.06\end{array}$ & $\begin{array}{r}48 \cdot 64 \\
0-07 \\
0-29 \\
0-04 \\
0-00 \\
45 \cdot 10 \\
0-29 \\
6.59 \\
0-48 \\
0-00 \\
101.52\end{array}$ \\
\hline $\begin{array}{l}\mathrm{Si}^{i \mathbf{V}} \\
\mathrm{Al}^{\mathrm{IV}} \\
\mathrm{Al}^{\mathrm{VI}} \\
\mathrm{Ti} \\
\mathrm{Cr} \\
\mathrm{Fe}^{3+} \\
\mathrm{Fe}^{2+} \\
\mathrm{Mn} \\
\mathrm{Mg} \\
\mathrm{Ca} \\
\mathrm{Na}\end{array}$ & $\begin{array}{l}1.99 \\
0-01 \\
0-01 \\
0-00 \\
0-00 \\
0-00 \\
1.56 \\
0-00 \\
0-39 \\
0-02 \\
0-00\end{array}$ & $\begin{array}{l}2-00 \\
0-00 \\
0-02 \\
0-00 \\
0-00 \\
0-00 \\
1.55 \\
0-01 \\
0-41 \\
0-02 \\
0-00\end{array}$ & $\begin{array}{l}1.99 \\
0-01 \\
0-01 \\
0-00 \\
0-00 \\
0-01 \\
1.54 \\
0-01 \\
0-41 \\
0-03 \\
0-00\end{array}$ & $\begin{array}{l}201 \\
0-00 \\
0-01 \\
0-00 \\
0-00 \\
0-00 \\
1.55 \\
0-01 \\
0-40 \\
0-02 \\
0-00\end{array}$ & $\begin{array}{l}2-00 \\
0-01 \\
0-01 \\
0-00 \\
0-00 \\
0-00 \\
1.50 \\
0-01 \\
0-44 \\
0-03 \\
0-00\end{array}$ & $\begin{array}{l}1.97 \\
0-02 \\
0-00 \\
0-00 \\
0-00 \\
0-04 \\
1.47 \\
0-01 \\
0-47 \\
0-02 \\
0-00\end{array}$ & $\begin{array}{l}2.00 \\
0-00 \\
0-01 \\
0-00 \\
0-00 \\
0-00 \\
1.58 \\
0-01 \\
0-37 \\
0-03 \\
0-00\end{array}$ & $\begin{array}{l}1.98 \\
0-02 \\
0-00 \\
0-00 \\
0-00 \\
0-02 \\
1.55 \\
0-01 \\
0-40 \\
0-03 \\
0-00\end{array}$ & $\begin{array}{l}2-00 \\
0-00 \\
0-00 \\
0-00 \\
0-00 \\
0-00 \\
1.63 \\
0-02 \\
0-30 \\
0-04 \\
0-00\end{array}$ & $\begin{array}{l}2-02 \\
0-00 \\
0-01 \\
0-00 \\
0-00 \\
0-00 \\
1.61 \\
0-02 \\
0-30 \\
0-04 \\
0-00\end{array}$ & $\begin{array}{l}1.99 \\
0-01 \\
0-00 \\
0-00 \\
0-00 \\
0-01 \\
1.61 \\
0-01 \\
0-33 \\
0-02 \\
0-00\end{array}$ & $\begin{array}{l}1.99 \\
0-01 \\
0-01 \\
0-00 \\
0-00 \\
0-00 \\
1.56 \\
0-01 \\
0-39 \\
0-03 \\
0-00\end{array}$ & $\begin{array}{l}2-00 \\
0-00 \\
0-01 \\
0-00 \\
0-00 \\
0-00 \\
1.55 \\
0-01 \\
0-40 \\
0-02 \\
0-00\end{array}$ \\
\hline $\begin{array}{l}X(\mathrm{Fs}) \\
X(\mathrm{En}) \\
a(\mathrm{Fs})\end{array}$ & $\begin{array}{l}0.79 \\
0-20 \\
0-61\end{array}$ & $\begin{array}{l}0.78 \\
0-21 \\
0-60\end{array}$ & $\begin{array}{l}0.77 \\
0-21 \\
0-60\end{array}$ & $\begin{array}{l}0.78 \\
0.20 \\
0-60\end{array}$ & $\begin{array}{l}0-76 \\
0-22 \\
0-56\end{array}$ & $\begin{array}{l}0-75 \\
0-24 \\
0-54\end{array}$ & $\begin{array}{l}0.79 \\
0.19 \\
0.62\end{array}$ & $\begin{array}{l}0-78 \\
0-20 \\
0-60\end{array}$ & $\begin{array}{l}0-82 \\
0-15 \\
0-68\end{array}$ & $\begin{array}{l}0-82 \\
0-15 \\
0-67\end{array}$ & $\begin{array}{l}0-82 \\
0-17 \\
0-66\end{array}$ & $\begin{array}{l}0.78 \\
0-20 \\
0-61\end{array}$ & $\begin{array}{l}0.78 \\
0-20 \\
0.60\end{array}$ \\
\hline
\end{tabular}

Normalization: four cations. Core and rim activities $a(\mathrm{Fs})=a\left(\mathrm{Fe}_{2} \mathrm{Si}_{2} \mathrm{O}_{6}\right)$ at 750 and $650^{\circ} \mathrm{C}$, respectively. Fs $=$ ferrosilite, En $=$ enstatite, n.d. $=$ not determined. 
TABLE 5

Representative plagioclase analyses

\begin{tabular}{|c|c|c|c|c|c|c|c|c|c|c|c|}
\hline & $\begin{array}{c}\text { A22-2 } \\
\text { core }\end{array}$ & $\begin{array}{c}\text { W24-1 } \\
\text { core }\end{array}$ & $\begin{array}{l}\text { W58-1 } \\
\text { core }\end{array}$ & $\underset{\text { rim }}{\text { H58-I }}$ & $\begin{array}{l}\text { W58-4 } \\
\text { core }\end{array}$ & $\begin{array}{c}\text { W58-4 } \\
\text { rim }\end{array}$ & $\begin{array}{l}T 2-E \\
\text { core }\end{array}$ & $\begin{array}{l}\text { T2-E } \\
\text { rim }\end{array}$ & $\begin{array}{c}\text { T2-4-2 } \\
\text { core }\end{array}$ & $\begin{array}{l}T 7-12 \\
\text { core }\end{array}$ & $\underset{\mathrm{rim}}{\mathrm{T}-12}$ \\
\hline $\begin{array}{l}\mathrm{SiO}_{2} \\
\mathrm{Al}_{2} \mathrm{O}_{3} \\
\mathrm{CaO} \\
\mathrm{Na}_{2} \mathrm{O} \\
\mathrm{K}_{2} \mathrm{O} \\
\mathrm{Sum}\end{array}$ & $\begin{array}{r}57.59 \\
27.84 \\
9.33 \\
6.07 \\
0-08 \\
100-91\end{array}$ & $\begin{array}{r}60-93 \\
23 \cdot 27 \\
5 \cdot 34 \\
10-19 \\
0-10 \\
99 \cdot 84\end{array}$ & $\begin{array}{r}57 \cdot 46 \\
25 \cdot 20 \\
7 \cdot 32 \\
9 \cdot 11 \\
0-11 \\
99 \cdot 20\end{array}$ & $\begin{array}{r}58 \cdot 48 \\
24.69 \\
7.08 \\
8.82 \\
0.17 \\
99.23\end{array}$ & $\begin{array}{r}57.79 \\
24.89 \\
7.67 \\
8.68 \\
0.23 \\
99.26\end{array}$ & $\begin{array}{r}57.84 \\
25.06 \\
7.47 \\
8.59 \\
0.13 \\
99.09\end{array}$ & $\begin{array}{r}60-03 \\
24.64 \\
6.90 \\
7.35 \\
0.31 \\
99.23\end{array}$ & $\begin{array}{r}58.92 \\
24.37 \\
7.23 \\
7.62 \\
0.20 \\
98.33\end{array}$ & $\begin{array}{r}60-67 \\
25.67 \\
6.70 \\
7.46 \\
0.32 \\
100.82\end{array}$ & $\begin{array}{r}60-89 \\
23 \cdot 93 \\
6 \cdot 48 \\
8 \cdot 18 \\
0-06 \\
99 \cdot 53\end{array}$ & $\begin{array}{r}61 \cdot 26 \\
24 \cdot 06 \\
6 \cdot 32 \\
8 \cdot 15 \\
0-03 \\
99 \cdot 82\end{array}$ \\
\hline $\begin{array}{l}\mathrm{Si} \\
\mathrm{Al} \\
\mathrm{Ca} \\
\mathrm{Na} \\
\mathrm{K}\end{array}$ & $\begin{array}{l}2.56 \\
1.46 \\
0.45 \\
0.52 \\
0.00\end{array}$ & $\begin{array}{l}2.67 \\
1.20 \\
0.25 \\
0.87 \\
0-01\end{array}$ & $\begin{array}{l}2.55 \\
1.32 \\
0.35 \\
0.78 \\
0-01\end{array}$ & $\begin{array}{l}2 \cdot 60 \\
1 \cdot 29 \\
0.34 \\
0-76 \\
0-01\end{array}$ & $\begin{array}{l}2.57 \\
1.30 \\
0.37 \\
0.75 \\
0-01\end{array}$ & $\begin{array}{l}2 \cdot 58 \\
1 \cdot 32 \\
0-36 \\
0-74 \\
0-01\end{array}$ & $\begin{array}{l}2 \cdot 70 \\
1 \cdot 31 \\
0-33 \\
0-64 \\
0-02\end{array}$ & $\begin{array}{l}2 \cdot 67 \\
1 \cdot 30 \\
0-35 \\
0-67 \\
0-01\end{array}$ & $\begin{array}{l}2 \cdot 69 \\
1 \cdot 34 \\
0-32 \\
0-64 \\
0-02\end{array}$ & $\begin{array}{l}2 \cdot 72 \\
1 \cdot 26 \\
0-31 \\
0-71 \\
0-00\end{array}$ & $\begin{array}{l}2 \cdot 73 \\
1 \cdot 26 \\
0-30 \\
0-70 \\
0-00\end{array}$ \\
\hline $\begin{array}{l}X(\text { An }) \\
a(\text { An })\end{array}$ & $\begin{array}{l}0-464 \\
0-611\end{array}$ & $\begin{array}{l}0-221 \\
0.289\end{array}$ & $\begin{array}{l}0-307 \\
0-422\end{array}$ & $\begin{array}{l}0-306 \\
0.474\end{array}$ & $\begin{array}{l}0.327 \\
0.439\end{array}$ & $\begin{array}{l}0.324 \\
0.504\end{array}$ & $\begin{array}{l}0.333 \\
0.448\end{array}$ & $\begin{array}{l}0-340 \\
0.524\end{array}$ & $\begin{array}{l}0-327 \\
0-430\end{array}$ & $\begin{array}{l}0.304 \\
0.420\end{array}$ & $\begin{array}{l}0.300 \\
0.478\end{array}$ \\
\hline
\end{tabular}

Normalization: five cations. Calculated activities of $\mathrm{CaAl}_{2} \mathrm{Si}_{2} \mathrm{O}_{8}[a(\mathrm{An})]$ in plagioclase at 750 and $650^{\circ} \mathrm{C}$ for core and rim, respectively. 
TABLE 6

Representative clinopyroxene analyses

\begin{tabular}{|c|c|c|c|c|c|c|c|c|c|c|}
\hline & $A / 6$ & $A 22-I$ & $A 22-2$ & W57-2 & W57-3 & $W 120-2$ & $W / 23$ & $T 1-4$ & $T 2.9$ & $T 2-10$ \\
\hline $\mathrm{SiO}_{2}$ & $49 \cdot 37$ & 48.95 & $48 \cdot 71$ & $48 \cdot 40$ & $48 \cdot 51$ & $51 \cdot 37$ & $51 \cdot 63$ & $51-08$ & $49 \cdot 48$ & $48 \cdot 72$ \\
\hline $\mathrm{TiO}_{2}$ & $0-00$ & $0-00$ & $0-04$ & $0-05$ & $0-01$ & $0-17$ & $0-21$ & n.d. & $0-01$ & n.d. \\
\hline $\mathrm{Al}_{2} \mathrm{O}_{3}$ & 1.08 & 100 & $1 \cdot 30$ & $1 \cdot 21$ & $1 \cdot 12$ & 1.67 & 1.61 & $0-10$ & $0-15$ & 0.20 \\
\hline $\mathrm{Cr}_{2} \mathrm{O}_{3}$ & $0-00$ & $0-00$ & $0-00$ & $0-01$ & $0-01$ & $0-04$ & $0-03$ & n.d. & $0-01$ & n.d. \\
\hline $\mathrm{Fe}_{2} \mathrm{O}_{3}$ & $0-51$ & $0-00$ & $2 \cdot 35$ & 1.56 & 1.24 & 2.91 & $1 \cdot 30$ & $0-00$ & 1.77 & $0-00$ \\
\hline $\mathrm{FeO}$ & $23 \cdot 35$ & $28 \cdot 74$ & $23 \cdot 37$ & $24 \cdot 51$ & $25 \cdot 30$ & 9.02 & 1065 & $24 \cdot 60$ & $24 \cdot 68$ & $26 \cdot 20$ \\
\hline $\mathrm{MnO}$ & $0-18$ & 0.18 & $0-19$ & $0-11$ & $0-07$ & $0-38$ & $0-28$ & $0-38$ & $0-24$ & $0-21$ \\
\hline $\mathrm{MgO}$ & $5 \cdot 18$ & $3 \cdot 24$ & $4 \cdot 19$ & 3.82 & 3.53 & $12 \cdot 46$ & $12 \cdot 56$ & 4.98 & $4 \cdot 26$ & $4 \cdot 41$ \\
\hline $\mathrm{CaO}$ & $20-51$ & 17.99 & $20-01$ & 19.91 & 19.99 & $21 \cdot 75$ & $21 \cdot 19$ & $20-61$ & $20-29$ & $21 \cdot 14$ \\
\hline $\mathrm{Na}_{2} \mathrm{O}$ & $0-00$ & $0-00$ & $0-34$ & $0-20$ & $0-15$ & $0-44$ & 0.30 & n.d. & 0.14 & n.d. \\
\hline Sum & 100.17 & $100-22$ & $100-52$ & 99.81 & 99.94 & $100-24$ & $99-78$ & $101 \cdot 76$ & 101.04 & $100-88$ \\
\hline $\mathrm{Si}^{\mathbf{I V}}$ & 1.97 & 1.99 & 1.95 & 1.95 & 1.96 & 1.93 & 1.95 & 2.01 & 1.97 & 1.94 \\
\hline $\mathrm{Al}^{\mathrm{IV}}$ & $0-03$ & $0-01$ & 005 & $0-05$ & 004 & $0-07$ & 005 & $0-00$ & $0-01$ & $0-01$ \\
\hline$A l^{\mathrm{V}}$ & $0-02$ & $0-04$ & $0-01$ & $0-01$ & $0-01$ & $0-01$ & $0-02$ & $0-00$ & $0-00$ & 000 \\
\hline $\mathrm{Ti}$ & $0-00$ & $0-00$ & $0-00$ & $0-00$ & $0-00$ & $0-00$ & $0-01$ & n.d. & $0-00$ & n.d. \\
\hline $\mathrm{Cr}$ & $0-00$ & $0-00$ & $0-00$ & $0-00$ & $0-00$ & $0-00$ & 000 & n.d. & $0-00$ & n.d. \\
\hline $\mathrm{Fe}^{3+}$ & $0-02$ & $0-00$ & $0-07$ & $0-05$ & $0-04$ & $0-08$ & 004 & $0-00$ & $0-05$ & $0-00$ \\
\hline $\mathrm{Fe}^{2+}$ & 0.78 & 0.98 & $0-78$ & $0-83$ & $0-86$ & $0-28$ & $0-34$ & 081 & $0-82$ & 087 \\
\hline Mn & $0-01$ & 001 & $0-01$ & $0-00$ & $0-00$ & $0-01$ & 001 & $0-01$ & $0-01$ & 001 \\
\hline $\mathbf{M g}$ & $0-31$ & $0-20$ & 0.25 & 0.23 & 0.21 & $0-70$ & 071 & $0-29$ & $0-25$ & $0-26$ \\
\hline $\mathrm{Ca}$ & $0-88$ & 0.78 & $0-86$ & $0-86$ & 0.87 & $0-88$ & $0-86$ & 0.87 & $0-87$ & 0.90 \\
\hline $\mathrm{Na}$ & $0-00$ & $0-00$ & $0-03$ & $0-02$ & $0-01$ & $0-03$ & $0-02$ & n.d. & $0-01$ & n.d. \\
\hline
\end{tabular}

Normalization: four cations. All formulae are reintegrated from 100 to 200 spot analyses.

TABLE 7

Representative cordierite analyses

\begin{tabular}{|c|c|c|c|c|c|c|}
\hline & $\begin{array}{c}\text { W12l-l } \\
\text { late }\end{array}$ & $\begin{array}{c}W 121-1 \\
\text { incl }\end{array}$ & $W / 21-4$ & $W / 40-I$ & $w 140-2$ & $w / 40-5$ \\
\hline $\mathrm{SiO}_{2}$ & 48.47 & $49-02$ & $48 \cdot 70$ & 48.43 & $48 \cdot 25$ & 47.93 \\
\hline $\mathrm{TiO}_{2}$ & $0-04$ & $0-02$ & $0-00$ & $0-01$ & $0-00$ & $0-00$ \\
\hline $\mathrm{Al}_{2} \mathrm{O}_{3}$ & $33-08$ & $32 \cdot 64$ & $32 \cdot 47$ & $33 \cdot 31$ & $33-04$ & 3302 \\
\hline $\mathrm{Cr}_{2} \mathrm{O}_{3}$ & $0-01$ & $0-00$ & $0-01$ & $0-00$ & $0-00$ & 000 \\
\hline $\mathrm{Fe}_{2} \mathrm{O}_{3}$ & $0-00$ & 1.42 & 1.43 & 1.69 & 064 & 1.92 \\
\hline $\mathrm{FeO}$ & 3.72 & 3.62 & 4.59 & 5.65 & 6.50 & 5.55 \\
\hline $\mathrm{MnO}$ & $0-05$ & $0-02$ & $0-06$ & $0-05$ & $0-00$ & $0-03$ \\
\hline $\mathrm{MgO}$ & 1069 & $10-84$ & $10-21$ & 8.95 & 8.87 & $8 \cdot 88$ \\
\hline $\mathrm{CaO}$ & $0-02$ & $0-05$ & $0-04$ & 005 & $0-02$ & 006 \\
\hline $\mathrm{Na}_{2} \mathrm{O}$ & $0-00$ & $0-08$ & $0-07$ & $0-24$ & $0-13$ & $0-21$ \\
\hline $\mathrm{K}_{2} \mathrm{O}$ & $0-00$ & $0-00$ & $0-00$ & 000 & $0-00$ & $0-01$ \\
\hline Sum & $96-08$ & $97 \cdot 71$ & 97.58 & $98 \cdot 38$ & 97.45 & 97.61 \\
\hline $\mathrm{H}_{2} \mathrm{O}, \mathrm{CO}_{2}^{*}$ & 3.92 & $2 \cdot 29$ & $2 \cdot 42$ & 1.62 & $2 \cdot 55$ & $2 \cdot 39$ \\
\hline $\mathrm{Si}$ & 500 & 4.99 & 4.99 & 4.95 & 4.98 & 4.94 \\
\hline $\mathrm{Ti}$ & $0-00$ & $0-00$ & $0-00$ & $0-00$ & $0-00$ & 000 \\
\hline Al & $4-02$ & 3.92 & 3.92 & 401 & $4-02$ & 401 \\
\hline $\mathrm{Cr}$ & $0-00$ & $0-00$ & $0-00$ & $0-00$ & $0-00$ & $0-00$ \\
\hline $\mathrm{Fe}^{3+}$ & $0-00$ & 0.11 & $0-11$ & 0.13 & $0-05$ & $0-15$ \\
\hline $\mathrm{Fe}^{2+}$ & $0-32$ & $0-31$ & $0-39$ & $0-48$ & 0.56 & $0-48$ \\
\hline $\mathbf{M n}$ & $0-00$ & $0-00$ & $0-01$ & $0-00$ & $0-00$ & 000 \\
\hline $\mathrm{Mg}$ & 1.64 & 1.65 & 1.56 & $1 \cdot 36$ & $1 \cdot 36$ & $1 \cdot 37$ \\
\hline $\mathrm{Ca}$ & $0-00$ & $0-01$ & $0-00$ & $0-01$ & $0-00$ & 001 \\
\hline $\mathrm{Ne}$ & $0-00$ & $0-02$ & $0-01$ & $0-05$ & $0-03$ & $0-04$ \\
\hline $\mathbf{K}$ & $0-00$ & $0-00$ & $0-00$ & 000 & 000 & 000 \\
\hline
\end{tabular}

* $\mathrm{H}_{2} \mathrm{O}, \mathrm{CO}_{2}$ content not measured; calculated to allow totals to sum to $100 \%$. Normalization: 11 cations. 

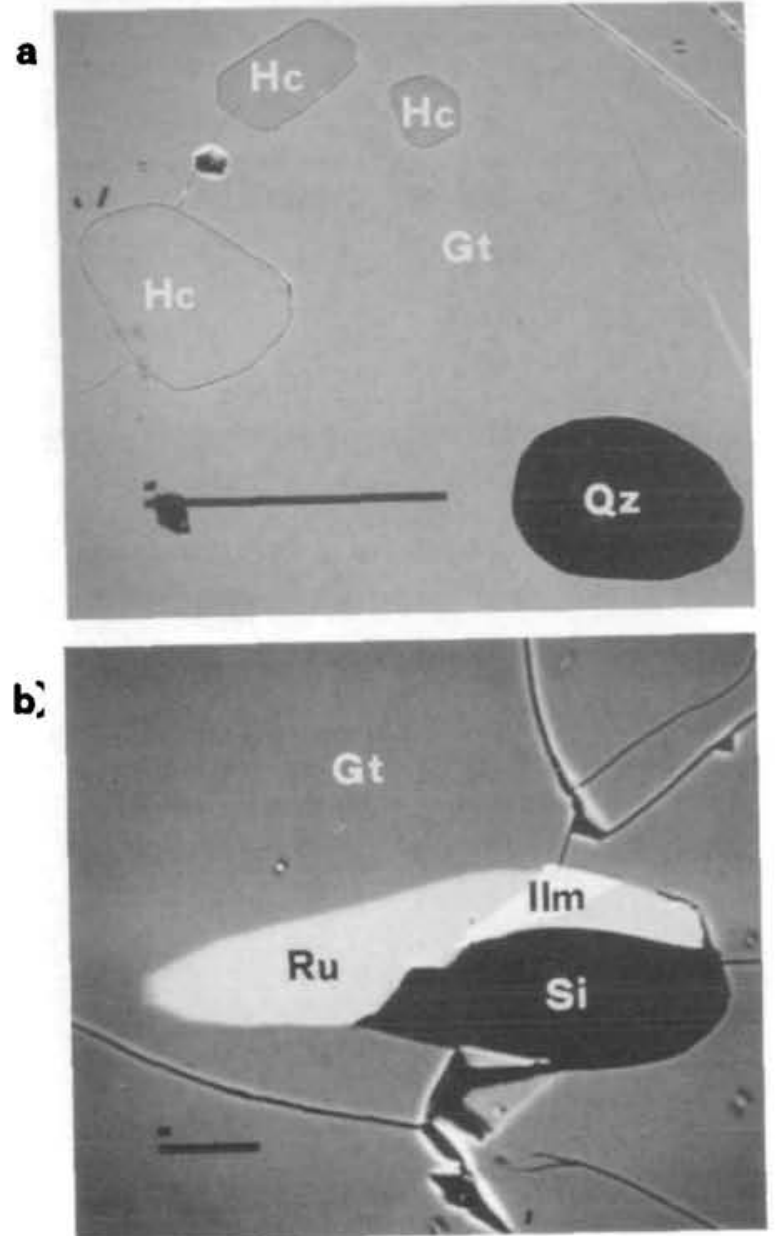

FIG. 2. Back-scattered image of inclusions in garnet. (a) Inclusions of quartz (Qz) and hercynite (Hc) in garnet (Gt) sample W78. Scale bar represents $100 \mu \mathrm{m}$. Zoning profile between the largest hercynite (center left) and quartz inclusion is presented in Fig. 4. (b) Inclusions of ilmenite ( $\mathrm{Im})$, rutile (Ru), and sillimanite (Si) in garnet sample W121. Scale bar represents $10 \mu \mathrm{m}$.

has an intermediate $\mathrm{d} P / \mathrm{d} T$ slope and is well calibrated (Bohlen et al., 1986). Isolated inclusions of the high-temperature products, hercynite and quartz, are occasionally found in the cores of garnets in samples that are otherwise spinel-free (Fig. 2a). These two phases are never found in contact with one another, presumably because they reacted to form garnet and sillimanite during retrogression. Hercynite could only be preserved if it became isolated from quartz by an early overgrowth of garnet. If quartz and hercynite were in equilibrium during this early garnet growth, the mineral grains, once isolated, could preserve chemistries corresponding to peak or near-peak metamorphic conditions. The spinel compositions within a single thin-section are all very similar (Table 2), supporting the argument that equilibrium was achieved between the included phases during the time of their entrapment. The lack of any gahnite-poor spinel outside of the garnet porphyroblasts is further evidence that the spinel is a high-temperature phase that is preserved only because it is isolated from quartz by the garnet overgrowths. 
The $\log _{10} K$ isopleths for reaction (1) were calculated from the relation

$$
\left.\Delta G\right|_{T_{2}} ^{P_{2}}-\left.\Delta G\right|_{T_{1}} ^{P_{1}}=\int_{P_{1}}^{P_{2}} \Delta V \mathrm{~d} P-\int_{T_{1}}^{T_{2}} \Delta S \mathrm{~d} T+R T \ln K
$$

where $K=a\left(\begin{array}{l}\left(\begin{array}{ll}\mathrm{Fe}_{3} \mathrm{Al}_{2} \mathrm{Si}_{3} \mathrm{O}_{12} \\ \mathrm{~F}_{12}\end{array}\right) / a\left(\mathrm{FeAl}_{2} \mathrm{O}_{4}\right. \\ \mathrm{Fercyn}_{4}\end{array}\right)^{3}$ and the activities of quartz and sillimanite are assumed to be unity (Fig. 3). The sources of the thermodynamic data needed for calculations are given in the caption of Fig. 3.

Minor retrograde $\mathrm{Mg}-\mathrm{Fe}$ exchange over a distance of $\sim 20 \mu \mathrm{m}$ is observed in the garnet away from spinel (Fig. 4) and no zoning is observed in the spinel. The lack of zoning in the spinel indicates that cation diffusion in spinel is more rapid than in garnet, and that any zoning profile that was present in the spinel at the blocking temperature of garnet was annealed by further intracrystalline diffusion. The closure temperature for cation diffusion in garnet can be approximated from available diffusion data for magnesium in garnet measured over the temperature interval $750-900^{\circ} \mathrm{C}$ (Cygan \& Lasaga, 1985). The approximate penetration distance can be calculated from the equation (Crank, 1975)

$$
x=\sqrt{ }(4 D t)
$$

where $x=$ distance, $D=$ diffusion coefficient at a particular temperature, and $t=$ duration.

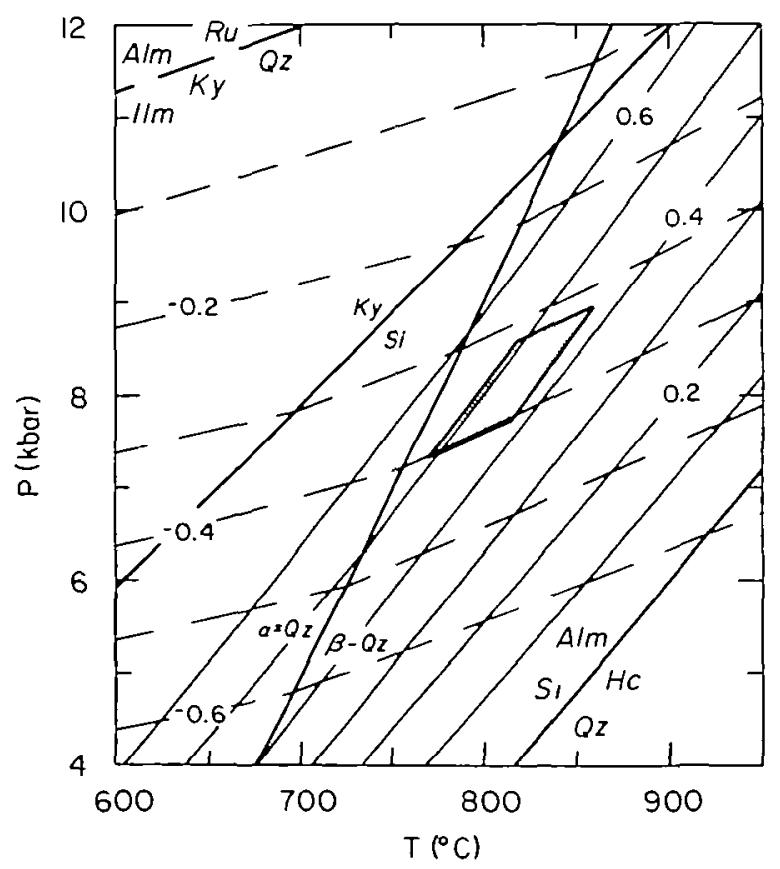

FIG. 3. $\log _{10} K$ isopleths for reactions (1) (solid) and (6) (dashed). Stippled field represents the intersections of reactions (1) and (6) adjusted for solid-solution in garnet and hercynite. Alm =almandine; Hc =hercynite; Ilm $=$ ilmenite; $\mathrm{Ky}=$ kyanite; $\mathrm{Ru}=$ rutile; $\mathrm{Si}=$ sillimanite; $\mathrm{Qz}=$ quartz. Sources of thermodynamic data: almandine: entropy $(S)-1$; volume $(V)-2$; expansivity $(\alpha)-2 ;$ compressibility $(\beta)-2 ;$ fayalite: $S-3 ; V-4 ; \alpha-5 ; \beta-4,5$; ferrosilite: $S-6 ; V, \alpha, \beta-4$; hercynite: $S, V, \alpha, \beta-7$; ilmenite: $S, V-8 ; \alpha-9 ; \beta-10$, kyanite: $S, V-11 ; \alpha-12 ; \beta-13$; quartz $S, V, \alpha, \beta-4$; rutile: $S, V-14 ; \alpha-15 ; \beta-16$; sillimanite. $S, V-11 ; \alpha-12 ; \beta-13$. References: (1) Metz et al. (1983) (2) Anovitz \& Essene (1987); (3) Robie et al. (1982) (4) Robinson et al. (1982), (5) Hazen (1976); (6) Bohlen et al. (1983ch) (7) Bohlen et al. (1986) (8) Anovitz et al. (1985) (9) assumed equal to hematite (data of Robinson et al, 1982), (10) Birch (1966); (11) Robie \& Hemingway (1984) (12) Winter \& Ghose (1979); (13) Brace et al. (1969); (14) Robie et al. (1978) (15)-Meagher \& Lager (1979); (16) Hazen \& Finger (1981). 


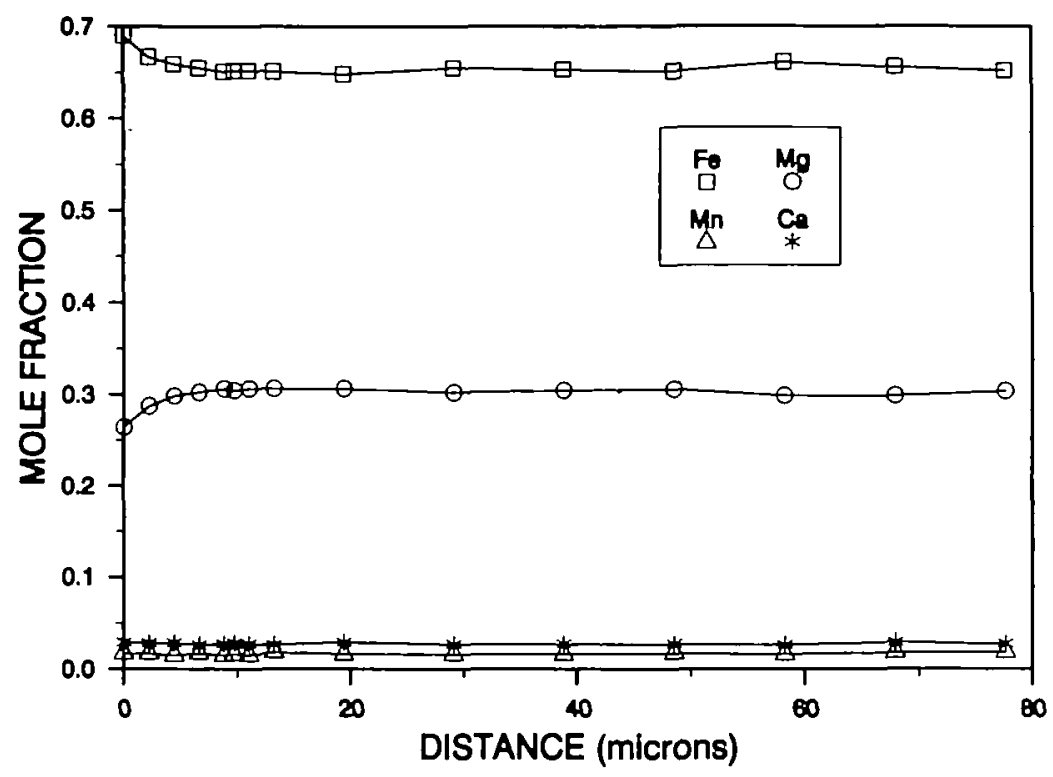

FIG. 4. Zoning profile in garnet between spinel (at $0 \mu \mathrm{m}$ ) and quartz (at $78 \mu \mathrm{m}$ ) in sample W78.

The observed penetration distance of $20 \mu \mathrm{m}$ will take $10 \mathrm{Ma}$ at a temperature of $480^{\circ} \mathrm{C}$ $\left(\log _{10} D\left(\mathrm{~m}^{2} / \mathrm{s}\right)=-24 \cdot 6\right)$ and $1 \mathrm{Ma}$ at a temperature of $520^{\circ} \mathrm{C}\left(\log _{10} D=-23 \cdot 8\right)$. Alternatively, the closure temperature can be estimated from the following equation (Dodson, 1973):

$$
\frac{Q}{R T_{\mathrm{c}}}=\ln \left(-\frac{A R T_{\mathrm{c}}^{2} D_{\mathrm{o}}}{a^{2} Q s}\right)
$$

where $Q=$ activation energy $(239 \mathrm{~kJ} / \mathrm{mol}), D_{0}=$ pre-exponential factor $\left(9 \cdot 8 \times 10^{-9} \mathrm{~m}^{2} / \mathrm{s}\right)$, $A=$ geometric factor ( 55 for a sphere, 8.7 for a plane), $T_{\mathrm{c}}=$ closure temperature (in ${ }^{\circ} \mathrm{C}$ ), $a=$ grain radius and $s=$ cooling rate. Assuming a linear cooling rate of $1-10^{\circ} \mathrm{C} / \mathrm{Ma}$ (Berger \& York, 1981; Cosca et al., 1991), a grain radius of $100 \mu \mathrm{m}$ and planar geometry at the spinel-garnet interface, the closure temperature will be 520 and $480^{\circ} \mathrm{C}$ for a cooling rate of 10 and $1{ }^{\circ} \mathrm{C} / \mathrm{Ma}$, respectively. The $\mathrm{Mg}-\mathrm{Fe}$ self-diffusion rate in spinel must be significant below $500^{\circ} \mathrm{C}$ in a slowly cooled metamorphic terrane.

To calculate maximum temperatures from the assemblage spinel + quartz, the originahi... spinel and garnet compositions must be determined. It was assumed that the garnet far from the spinel interface did not significantly exchange with the spinel and represents the peaktemperature composition. The spinel composition can then be calculated by adding the iron lost from the spinel to the garnet during retrograde $\mathrm{Mg}-\mathrm{Fe}$ exchange back into the spinel. The $\mathrm{Fe}-\mathrm{Mg}$ diffusion profile in the garnet surrounding the spinel was assumed to be spherically symmetrical as both garnet and spinel are isometric. The $\mathrm{Fe} / \mathrm{Mg}$ diffusion profile in the garnet is measurable from the garnet interface to a distance of $14 \mu \mathrm{m}$ from the interface (Fig. 4). This profile was fitted with a simple third-order polynomial. The iron loss to the garnet was then calculated by integrating concentric 'shells' of constant $\mathrm{Fe}$ excess (mole fraction $\mathrm{Fe} / \mathrm{unit}$ volume) in the garnet using spherical coordinates:

$$
\text { Excess } \mathrm{Fe}=\int_{R}^{R+a} \int_{0}^{\pi} \int_{0}^{2 \pi} \rho^{2} \cdot\left(a+b x+c x^{2}+d x^{3}\right) \sin \phi \mathrm{d} \theta \mathrm{d} \phi \mathrm{d} \rho .
$$


$R$ is the radius of the spinel $(42 \mu \mathrm{m}), \alpha$ is the distance over which there is a measurable diffusion profile $(14 \mu \mathrm{m})$ and the term $\left(a+b x+c x^{2}+d x^{3}\right)$ is a third-order polynomial fitted to the measured $\mathrm{Fe} / \mathrm{Mg}$ diffusion profile in the garnet $(a=5 \cdot 748 ; b=-0.01159 ; c=0.001208$; $d=-4.174 \times 10^{-5} ; R^{2}=0.99$ ). The calculated peak-temperature spinel composition is only $0.009 \%$ more iron-rich than the measured composition. This small amount of exchange can safely be ignored, as it represents a compositional change that is far less than the errors in the microprobe determinations. However, for very small inclusions $(<10 \mu \mathrm{m})$, this type of postentrapment volume diffusion correction should have a measurable effect and should not be ignored.

Garnet-rutile-ilmenite-sillimanite-quartz equilibria. The reaction

$$
\begin{aligned}
& \mathrm{Fe}_{3} \mathrm{Al}_{2} \mathrm{Si}_{3} \mathrm{O}_{12}+3 \mathrm{TiO}_{2}=3 \mathrm{FeTiO}_{3}+\mathrm{Al}_{2} \mathrm{SiO}_{5}+2 \mathrm{SiO}_{2} \\
& \text { almandine rutile ilmenite sillimanitequartz }
\end{aligned}
$$

has a small positive $\mathrm{d} P / \mathrm{d} T$, making it an ideal geobarometer (Bohlen et al., 1983a). Rutile, ilmenite, sillimanite, and quartz inclusions are all found within single garnet porphyroblasts in the metapelitic units (Fig. 2b). No rutile has been found outside of garnet, however. Because rutile and garnet define the high-pressure assemblage of reaction (6), rutile inclusions in garnet will define a minimum pressure of equilibration at any temperature. For garnet and rutile to react, a volume increase of $11.6 \%$ (at $850^{\circ} \mathrm{C}, 8 \mathrm{~kb}$ ) is required. The volume occupied by a rutile inclusion in garnet is fixed, and so no expansion can take place during reaction. If the garnet remains rigid, then reaction (6) cannot take place. The beginning of rutile breakdown in garnet must result in either a pressure increase in the constant volume environment or the decrepitation of the garnet host. Large rutile grains will exert a greater pressure than small grains on the garnet host by reaction (6). This will rupture the garnet and allow rutile to completely react with garnet to form ilmenite, sillimanite, and quartz, consistent with a lack of large rutile grains in the garnet porphyroblasts. The strength of garnet at elevated temperatures and pressures is not well known, but the discovery of coesite in garnet porphyroblasts and its preservation during retrogression suggests that garnets can support large overpressures (Chopin, 1984). Therefore, rutile inclusions in garnet may provide the only quantitative evidence of peak metamorphic pressures.

Calculations of the stability field of reaction (6) at reduced activities (constant $\log _{10} K$ ) were generated from the experimental reversals of the metastable extension of reaction (6) in the kyanite field using the relation

$$
\int_{P_{1}}^{P_{2}} \Delta V \mathrm{~d} P=-R T \ln K
$$

where $K=a\left(\mathrm{FeTiO}_{3}\right)^{3} / a\left(\mathrm{Fe}_{\mathrm{F}_{3} \mathrm{Al}_{2} \mathrm{Si}_{3} \mathrm{O}_{12}}^{\mathrm{iln}}\right)$, and rutile, sillimanite, and quartz are assumed to have endmember compositions. The location of the kyanite equivalent of reaction (6) in the kyanite field was calculated by first determining the free energy for reaction (6) at the kyanite-sillimanite boundary and then calculating $\log _{10} K$ isopleths in the kyanite field using the $\Delta \mathrm{G}_{\text {reaction }}$ value at the kyanite-sillimanite boundary as a starting point. Thermodynamic data are given in the caption of Fig. 3. The calculated location of the $\log _{10} K$ isopleths (Fig. 3) are in agreement with those determined by Bohlen et al. (1983a).

\section{Combined pressure-temperature estimates from inclusion minerals in garnet}

Garnet compositions adjacent to rutile grains (Table 3) correspond to pressures of 8.0 $\pm 1.0 \mathrm{~kb}$ at $800^{\circ} \mathrm{C}$ and $7.0 \pm 1.0 \mathrm{~kb}$ at $600^{\circ} \mathrm{C}$ (Fig. 3). These are minimum pressures of equilibration, as the equilibrium location of reaction (6) is shifted to higher pressures for 
reduced activities of the phases ilmenite, sillimanite, and quartz. If the reduced activity (assuming ideal mixing) of ilmenite is considered (Samples W78-2, W121-1, Table 2), pressure estimates will be shifted to $\sim 9 \mathrm{~kb}$ at $800^{\circ} \mathrm{C}$. Measured garnet and spinel compositions (Tables 2 and 3) yield a $\log _{10} K$ of $0-48 \pm 0-04$ for reaction (1), corresponding to $700^{\circ} \mathrm{C}$ at $6.0 \mathrm{~kb}$ and $790^{\circ} \mathrm{C}$ at $8.0 \mathrm{~kb}$ (Fig. 3). The intersection of reactions (1) and (6), corrected for solid-solution, are at $8 \pm 1 \mathrm{~kb}$ and $815 \pm 50^{\circ} \mathrm{C}$.

\section{Pressures and temperatures of matrix minerals}

Temperatures and pressures for the matrix assemblages were estimated using wellcalibrated geothermometers and geobarometers. Different groundmass assemblages amenable to thermobarometric treatment were often found in different rock types. However, all rock types are geographically and structurally related, and have almost certainly experienced the same $P-T$ conditions.

\section{Garnet-pyroxene exchange thermometry}

Temperature estimates were made on matrix assemblages from coexisting garnet-clinopyroxene pairs in the iron formations and amphibolites, based on the calibrations of Ellis \& Green (1979) and Pattison \& Newton (1989). All clinopyroxenes have fine orthopyroxene lamellae. Clinopyroxene compositions (Table 6) were determined by averaging the sum of 40-100 analyses on a single grain with a beam rastered at $9 \mu \mathrm{m}^{2}$. Temperature estimates from Pattison \& Newton (1989) are $30-80^{\circ} \mathrm{C}$ lower than those of Ellis \& Green (1979) (Table 8). These lower temperatures are considered to be more reliable, as the calibration of Pattison \& Newton was made in the temperature range $700-1200^{\circ} \mathrm{C}$ and does not require a large extrapolation. The highest temperature estimate based on the calibration of Pattison \& Newton $\left(740^{\circ} \mathrm{C}\right)$ is considered to represent minimum temperatures, and garnet-clinopyroxene pairs recording lower temperature may have undergone retrograde resetting during cooling (Pattison \& Newton, 1989). Temperature estimates based on rim compositions are approximately $100^{\circ} \mathrm{C}$ lower than core temperatures (Table 8).

Garnet-orthopyroxene pairs are common in the northern Wind River iron formations. Temperatures were estimated for core and rim compositions of both garnet and orthopyroxene based on the equation of Lee \& Ganguly (1987) using a constant, non-zero $C_{\mathrm{p}}$ of reaction. Core compositions average $710 \pm 70^{\circ} \mathrm{C}$ and rim compositions average $605 \pm 35^{\circ} \mathrm{C}$ (Table 8). Actual uncertainties are probably greater than $50^{\circ} \mathrm{C}$, as the experimental calibration of Lee \& Ganguly had to be extrapolated $400^{\circ} \mathrm{C}$, but the temperature variations between core and rim should be more precise.

\section{Two-pyroxene solvus thermometry}

Orthopyroxene and clinopyroxene are commonly found together in the iron formations and less often in metabasites (hornblende granulites). Reintegrated clinopyroxene compositions were used for temperature estimates with the solvus of Lindsley (1983). The results yield scattered temperatures between 530 and $760^{\circ} \mathrm{C}$ (Table 8). The generally low temperatures are consistent with the concept of $\mathrm{Ca}-\mathrm{Mg}-\mathrm{Fe}^{2+}$ exchange during cooling (e.g., Essene, 1982). The use of the two-pyroxene thermometer of Davidson \& Lindsley (1985) results in temperature estimates approximately $25^{\circ} \mathrm{C}$ above those of Lindsley (1983). 
TABLE 8

Temperature estimates from garnet-clinopyroxene, garnet-orthopyroxene, and two-pyroxene thermometry

\begin{tabular}{|c|c|c|c|c|c|c|c|c|c|c|c|}
\hline \multirow[b]{2}{*}{ Sample } & & \multicolumn{3}{|c|}{ Garnet } & \multicolumn{3}{|c|}{ Orthopyroxene } & \multicolumn{3}{|c|}{ Clinopyroxene } & \multirow[b]{2}{*}{$\mathrm{TC} \dagger$} \\
\hline & & $\mathrm{X}(\mathrm{Ca})$ & $\mathrm{X}(\mathrm{Fe})$ & $X(M g)$ & $X(F e)$ & $\mathrm{X}\left(M_{g}\right)$ & $\mathrm{T}\left({ }^{\circ} \mathrm{C}\right)$ & $X(F e)$ & $\mathrm{X}(M g)$ & $T C^{\star}$ & \\
\hline A22-2 & (core) & 0.268 & $0-692$ & $0-023$ & 0.800 & $0-165$ & 530 & $0-440$ & 0.110 & 730 & 700 \\
\hline W24-1 & (core) & $0-093$ & $0-817$ & 0.093 & $0-670$ & $0-285$ & 650 & - & - & - & - \\
\hline$W 57-2$ & (core) & $0-247$ & 0.706 & $0-033$ & 0.825 & 0.145 & 730 & $0-415$ & $0-115$ & 775 & 705 \\
\hline W57-3 & (core) & 0.251 & 0.706 & $0-033$ & $0-840$ & 0.130 & 800 & 0.430 & 0.105 & 815 & 740 \\
\hline W58-1 & (core) & 0.143 & 0.787 & 0060 & 0.765 & $0-205$ & 710 & & & & \\
\hline W58-4 & (core) & 0.150 & 0.777 & $0-060$ & $0-780$ & $0-200$ & 745 & & & & \\
\hline W62-4 & (core) & $0-063$ & 0.787 & 0.147 & 0.545 & $0-415$ & 585 & & & & \\
\hline W62.5 & (core) & 0.063 & 0.793 & $0-140$ & $0-555$ & 0.405 & 580 & & & & \\
\hline T2-E-6 & (core) & 0.133 & 0.793 & $0-060$ & 0.775 & $0-200$ & 720 & & & & \\
\hline $\mathrm{T} 2-4-2$ & (core) & 0.137 & 0.793 & $0-057$ & 0.770 & 0.205 & 675 & & & & \\
\hline$T 2-4-7$ & (core) & 0.133 & 0.797 & $0-057$ & 0.755 & $0-255$ & 615 & & & & \\
\hline$T 2-6-1$ & (core) & 0.157 & 0.770 & $0-060$ & $0-750$ & $0-220$ & 680 & & & & \\
\hline T2-7-3 & (core) & $0-143$ & 0.793 & 0.047 & $0-790$ & $0-185$ & 645 & & & & \\
\hline$T 7.12$ & (core) & $0-133$ & 0.807 & $0-047$ & 0.787 & $0-160$ & 665 & & & & \\
\hline A22-2 & (rim) & 0.260 & 0.693 & $0-023$ & $0-800$ & 0.165 & 525 & $0-440$ & 0.110 & 720 & 675 \\
\hline W24-1 & (rim) & $0-087$ & 0.827 & $0-083$ & $0-670$ & $0-285$ & 590 & - & - & - & - \\
\hline W57-3 & (rim) & 0.240 & $0-723$ & $0-023$ & 0.830 & $0-140$ & 575 & $0-430$ & $0-105$ & 700 & 620 \\
\hline W58-1 & (rim) & 0.147 & 0.800 & $0-040$ & 0.775 & 0.200 & 535 & & & & \\
\hline W58-4 & (rim) & 0.177 & 0.773 & $0-040$ & $0-780$ & $0-200$ & 565 & & & & \\
\hline W62-4 & (rim) & $0-067$ & $0-817$ & 0.113 & 0.545 & $0-415$ & 470 & & & & \\
\hline T2-E-6 & (rim) & 0.130 & $0-810$ & $0-047$ & $0-765$ & $0-210$ & 560 & & & & \\
\hline $\mathrm{T} 2-4-2$ & (rim) & 0.140 & $0-800$ & $0-040$ & 0.775 & $0-200$ & 535 & & & & \\
\hline T2-6-1 & (rim) & 0.183 & 0.763 & $0-040$ & 0.735 & $0-235$ & 495 & & & & \\
\hline $\mathrm{T} 2.7-3$ & (rim) & 0.153 & 0.793 & $0-043$ & 0.775 & $0-200$ & 575 & & & & \\
\hline \multirow[t]{2}{*}{$\mathrm{T} 7-12$} & (rim) & $0-134$ & $0-813$ & $0-041$ & $0-781$ & $0-204$ & 590 & & & & \\
\hline & \multicolumn{7}{|c|}{ Opx/Cpx temperature estimates (based on $C p x$ compositions) } & & & & \\
\hline Sample & \multicolumn{2}{|c|}{$\mathrm{X}$ (Wollastonite $)$} & \multicolumn{2}{|c|}{$\mathrm{X}($ Enstatite $)$} & \multicolumn{2}{|c|}{$\mathrm{X}($ Ferrosilite $)$} & $\mathrm{T}\left({ }^{\circ} \mathrm{C}\right)$ & & & & \\
\hline A $16-4$ & \multicolumn{2}{|c|}{$0-428$} & \multicolumn{2}{|c|}{$0-163$} & \multicolumn{2}{|c|}{0.409} & 630 & & & & \\
\hline A22-1 & \multicolumn{2}{|c|}{0.374} & \multicolumn{2}{|c|}{$0-105$} & \multicolumn{2}{|c|}{$0-522$} & 760 & & & & \\
\hline A $22-2$ & \multicolumn{2}{|c|}{$0-388$} & \multicolumn{2}{|c|}{$0-121$} & \multicolumn{2}{|c|}{$0-491$} & 720 & & & & \\
\hline W57-2 & \multicolumn{2}{|c|}{$0-432$} & \multicolumn{2}{|c|}{$0-123$} & \multicolumn{2}{|c|}{$0-444$} & 580 & & & & \\
\hline W57-3 & \multicolumn{2}{|c|}{$0-432$} & \multicolumn{2}{|c|}{$0-113$} & & & 580 & & & & \\
\hline W120-2 & & & & & & & 630 & & & & \\
\hline T1-4 & & & & & & & 530 & & & & \\
\hline T2-9-6 & & & & & & & 530 & & & & \\
\hline $\mathrm{T} 2-10$ & & & & & & & 580 & & & & \\
\hline
\end{tabular}

* $T\left({ }^{\circ} \mathrm{C}\right)$ from Ellis \& Green (1979).

$\dagger T\left({ }^{\circ} \mathrm{C}\right)$ from Pattison \& Newton (1989). 

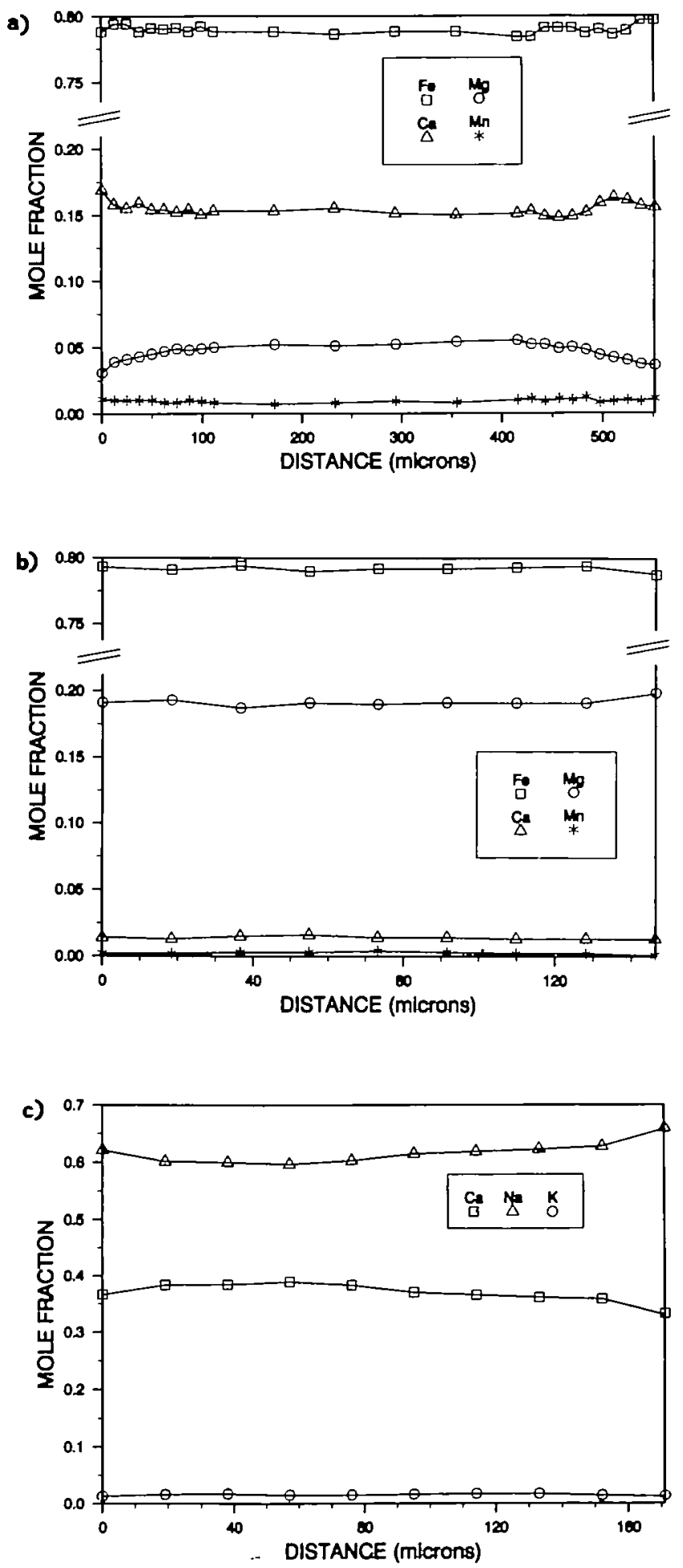

Fig. 5. Zoning profiles in garnet (a), orthopyroxene (b), and plagioclase (c) in W58. Profiles bounded by the following minerals: garnet by orthopyroxene; orthopyroxene by garnet; plagioclase by orthopyroxene. 


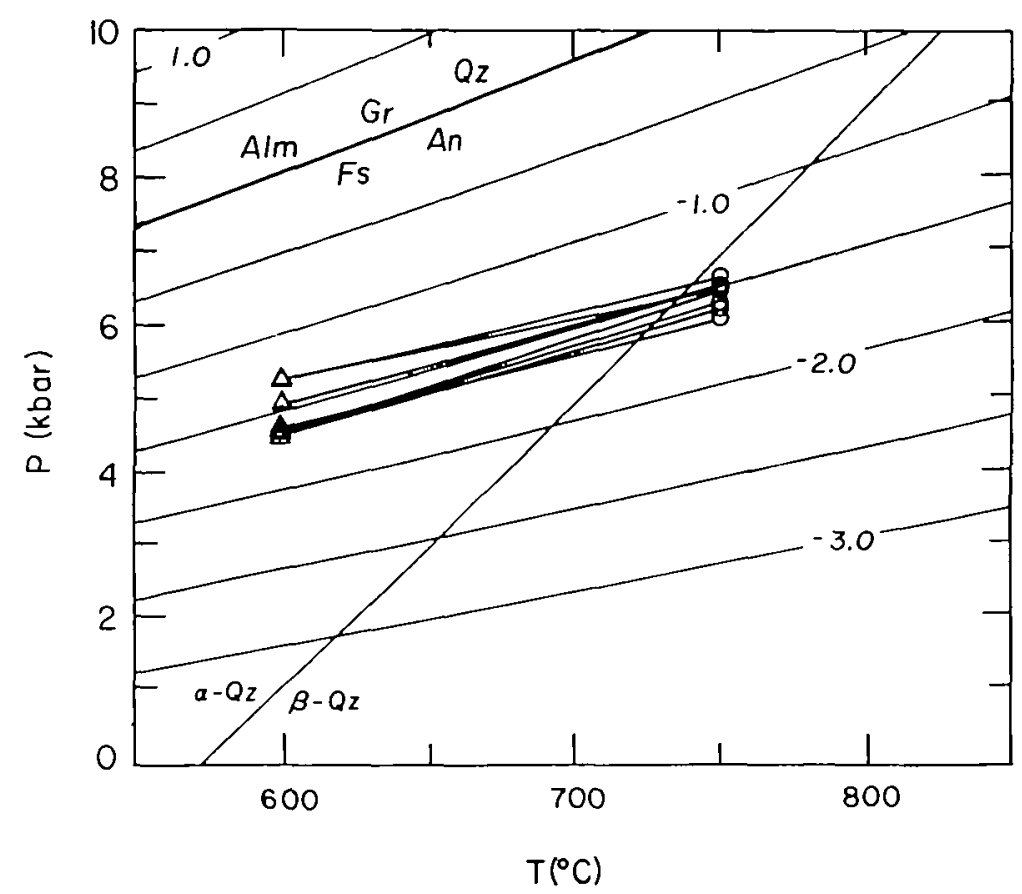

FIG. 6. $\log _{10} K$ isopleths for reaction (8). Connected circles and triangles represent core and rim compositions, respectively, of individual samples corrected for solid-solutions. Gr m grossular, Alm =almandine; $Q z=q u a r t z$, An $=$ anorthite; Fs m ferrosilite.

\section{Garnet-quartz-plagioclase-orthopyroxene barometry}

The assemblage garnet-quartz-plagioclase-orthopyroxene is common in the iron formations of the northern Wind River Range. The reaction

$$
\begin{array}{ll}
\mathrm{Ca}_{3} \mathrm{Al}_{2} \mathrm{Si}_{3} \mathrm{O}_{12}+2 \mathrm{Fe}_{3} \mathrm{Al}_{2} \mathrm{Si}_{3} \mathrm{O}_{12}+3 \mathrm{SiO}_{2}=3 \mathrm{CaAl}_{2} \mathrm{Si}_{2} \mathrm{O}_{8}+6 \mathrm{FeSiO}_{3} \\
\text { grossular almandine quartz anorthite ferrosilite }
\end{array}
$$

is a sensitive geobarometer as a result of the large $\Delta V_{\text {reaction }}$. The $P-T$ location of this reaction has been calculated by Bohlen et al. (1983b), and revised by Anovitz \& Essene (1987) based on a modified solution model for the unmixed garnet. Core and rim analyses of all phases (Tables 3-5) and zoning profiles of all phases in selected samples were determined (Fig. 5). Pressure estimates from core compositions are $6.0-6.7 \mathrm{~kb}$ at $750^{\circ} \mathrm{C}$. Pressure estimates from rim compositions, correcting activities for temperature, are $5.0-5.7 \mathrm{~kb}$ at $600^{\circ} \mathrm{C}$. This is consistent with a nearly isobaric cooling path over this $150^{\circ} \mathrm{C}$ interval (Fig. 6), as noted by Bohlen (1987) for other granulite terranes.

Ferrosilite-fayalite-quartz barometry

The reaction

$$
\underset{\text { ferrosilite fayalite quartz }}{\mathrm{Fe}_{2} \mathrm{Si}_{2} \mathrm{O}_{6}}=\underset{\mathrm{Fe}_{2} \mathrm{SiO}_{4}}{\mathrm{SiO}_{2}}
$$


has been calibrated by Bohlen et al. (1980) and Bohlen \& Boettcher (1981) for various dilutions of $\mathrm{Mg}$ for $\mathrm{Fe}$. The exchange reaction

$$
\mathrm{Fe}_{2} \mathrm{Si}_{2} \mathrm{O}_{6}+\mathrm{Mg}_{2} \mathrm{SiO}_{4}=\mathrm{Mg}_{2} \mathrm{Si}_{2} \mathrm{O}_{6}+\mathrm{Fe}_{2} \mathrm{SiO}_{4}
$$

ferrosilite forsterite enstatite fayalite

buffers the $\mathrm{Mg}-\mathrm{Fe}$ ratio between coexisting orthopyroxene and olivine at any temperature, so that the composition of orthopyroxene coexisting with olivine and quartz uniquely defines the pressure at a given temperature. Bohlen \& Boettcher (1981) have determined univariant curves of constant olivine and orthopyroxene composition for reaction (9). Pressure estimates at $750^{\circ} \mathrm{C}$ are $4.5 \mathrm{~kb}$ from the orthopyroxene isopleths and $6.5 \mathrm{~kb}$ from the olivine isopleths. To evaluate the apparent discrepancy between these two calibrations, $\log _{10} K$ isopleths for reaction (9) were generated from the endmember reaction using equation (7), where $K=\left\{a\left(\begin{array}{c}(\text { ayalite } \\ \left.\mathrm{Fe}_{2} \mathrm{SiO}_{4}\right)\end{array}\right) /\left\{a\left(\underset{\mathrm{Fe}_{2} \mathrm{~S}_{2} \mathrm{O}_{6}}{\text { ferrilite }}\right)\right\}\right.$. At constant temperature, the calculated location of $\log _{10} K$ isopleths at different pressures is a function only of the volume change of reaction. Thermodynamic data are given in the caption of Fig. 3. The excess volume of $\mathrm{Mg}-\mathrm{Fe}$ exchange in olivine and pyroxene was assumed to be zero. Pressure estimates for coexisting quartz, ferrosilite, and fayalite (Tables 2 and 4 ) are $4.8-5.4 \mathrm{~kb}$ (Fig. 7), lower than the upper pressure estimates from reaction (8). The discrepancy between the pressure estimates using the orthopyroxene and olivine isopleths of Bohlen \& Boettcher (1981) may be due to a lack of simultaneous equilibrium for reactions (9) and (10) during the experimental runs. Nucleation of an orthopyroxene from olivine and quartz with a composition only slightly different from the equilibrium composition dictated by reaction (10) can dramatically affect the location of reaction (9) for different composition isopleths. Alternatively, partial retrograde $\mathrm{Fe}-\mathrm{Mg}$ diffusional exchange in slowly cooled natural systems may occur by the temperature-sensitive reaction (10). Olivine, orthopyroxene, and quartz would then be slightly out of equilibrium, if the pressure-sensitive reaction (9) is more

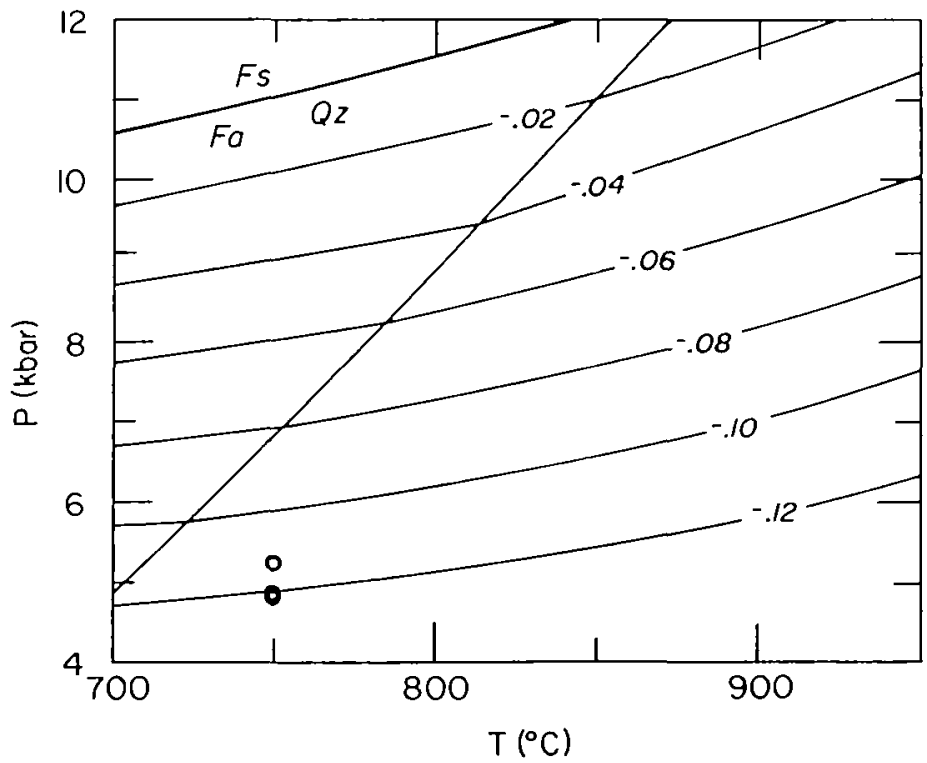

FIG. 7. $\log _{10} K$ isopleths for reaction (9). Circles represent calculated pressures corrected for solid-solution. $\mathrm{Fa}=$ fayalite; $\mathrm{Qz}=$ quartz; $\mathrm{Fs}=$ ferrosilite. 
sluggish at the retrograde temperatures. The large pressure shifts that result from only minor changes in composition make the ferrosilite-fayalite-quartz geobarometer too sensitive for accurate pressure estimates. Even minor cation exchange during cooling will dramatically affect the calculated pressure using reaction (9). Until the above discrepancies are properly evaluated, the $\log _{10} K$ method based on the endmember reaction (Bohlen et al., 1980) is best used as a check on other geobarometers.

\section{Garnet-cordierite equilibria}

The large partitioning of iron and magnesium between cordierite and garnet has been used alone, or in conjunction with buffering reactions, as a method of constraining metamorphic pressures and temperatures of cordierite-bearing assemblages. The accuracy of these thermobarometers has been limited by the complex effect of structural water and possible effects of order-disorder (e.g., Newton, 1972; Newton \& Wood, 1979). As a result, different experimental and thermodynamic stability relations have been determined involving cordierite stability, and the use of any equilibria involving cordierite can only be regarded as semi-quantitative (Essene, 1989). Temperatures and pressures based on $\mathbf{M g}-\mathrm{Fe}$ partitioning between cordierite and garnet and the reaction

$$
\begin{array}{lrr}
3\left(\mathrm{Mg}, \mathrm{Fe}_{2}\right)_{2} \mathrm{Al}_{4} \mathrm{Si}_{5} \mathrm{O}_{18}= & 2(\mathrm{Mg}, \mathrm{Fe})_{3} \mathrm{Al}_{2} \mathrm{Si}_{3} \mathrm{O}_{12}+\underset{\text { cordierite }}{4 \mathrm{Al}_{2} \mathrm{SiO}_{5}}+\underset{\text { sillimanite }}{5 \mathrm{SiO}_{2}} \\
& \text { quartz }
\end{array}
$$

were made using various calibrations (Thompson, 1976; Newton \& Wood, 1979; Martignole \& Sisi, 1981; Aranovich \& Podlesskii, 1983; Perchuk \& Lavrent'eva, 1983).

Temperature estimates based on $\mathrm{Mg}-\mathrm{Fe}$ exchange are varied and generally low (Table 9). Temperature estimates from core and rim compositions are $550-610$ and $\sim 430-585^{\circ} \mathrm{C}$. The low apparent temperatures are consistent with continued $\mathbf{M g}-\mathrm{Fe}$ exchange between various phases during retrogression. In particular, the phases cordierite and biotite (ubiquitous in all cordierite-bearing assemblages in the Wind River Range) probably continued to exchange during cooling below the blocking temperature of garnet.

Combined $P-T$ estimates were constrained based on reaction (11). Pressure and temperature estimates are in the range $4.8-6.2 \mathrm{~kb}, 570-630^{\circ} \mathrm{C}$, for core compositions, and $\sim 2.6-8.0 \mathrm{~kb}, 450-570^{\circ} \mathrm{C}$, for rim compositions, except with the calibration of Newton \& Wood (1979), for which there are no $P-T-a\left(\mathrm{H}_{2} \mathrm{O}\right)$ conditions that correspond to the $\mathrm{Mg} /\left(\mathrm{Mg}+\mathrm{Fe}^{2+}\right)$ ratios in both the garnet and cordierite (Table 9). The $P-T$ estimates from the rim compositions are especially varied. The extreme scatter may be due to the large extrapolations required to use the garnet-cordierite thermometers and to the late $\mathbf{M g}-\mathrm{Fe}$ exchange that is expected between garnet and cordierite.

\section{Comparison with temperature estimates from isotope thermometry}

Sharp et al. (1988) estimated metamorphic temperatures for the northern Wind River Range based on the oxygen isotope fractionations between minerals in the banded iron formations. They found that there was a spatially controlled variation in the isotopic fractionations between minerals as a function of the proximity to the surrounding orthogneisses, controlled by the degree of retrograde deformation of the sample. In this study, cation-based thermometers have been applied to some of the same samples studied by Sharp et al. (1988), to evaluate the relative retentivities of cation- and anion-based thermometers. 
TABLE 9

Temperature estimates from garnet-cordierite equilibria and exchange thermometry

\begin{tabular}{|c|c|c|c|c|c|c|c|c|c|c|c|}
\hline \multirow{2}{*}{\multicolumn{2}{|c|}{ Sample }} & \multirow[b]{2}{*}{$\mathrm{X}(\mathrm{Ca})$} & \multirow{2}{*}{$\begin{array}{c}\text { Garnet } \\
\mathrm{X}(F e)\end{array}$} & \multirow[b]{2}{*}{$X(M g)$} & \multicolumn{2}{|c|}{ Cordierite } & \multirow[b]{2}{*}{$T 1$} & \multirow[b]{2}{*}{$T 2$} & \multirow[b]{2}{*}{$P T 3$} & \multirow[b]{2}{*}{ PT4 } & \multirow[b]{2}{*}{ PT5 } \\
\hline & & & & & $X(F e)$ & $\mathrm{X}\left(M_{g}\right)$ & & & & & \\
\hline W121-4* & (core) & $0-027$ & $0-709$ & $0-251$ & $0-242$ & $0-751$ & 570 & 610 & $630 / 6-5^{*}$ & $630 / 5 \cdot 3^{*}$ & $570 / 5 \cdot 8$ * \\
\hline W140-1 & (core) & $0-034$ & 0.775 & 0.178 & $0-297$ & $0-671$ & 550 & 580 & $600 / 6 \cdot 2$ & $620 / 4 \cdot 7$ & $620 / 6 \cdot 0^{*}$ \\
\hline W140-2 & (core) & $0-027$ & 0.775 & 0.185 & $0-310$ & $0-682$ & 550 & 600 & $610 / 6 \cdot 2$ & $630 / 6 \cdot 3$ & $620 / 60$ \\
\hline$W 140-5$ & (core) & $0-030$ & 0.773 & 0.180 & 0.312 & $0-668$ & 560 & 600 & $610 / 6 \cdot 1$ & $640 / 4.8$ & $630 / 6 \cdot 2$ \\
\hline$W 121-1^{*}$ & (late) & $0-027$ & 0.623 & $0-337$ & $0-162$ & 0.840 & 530 & 585 & $500 / 8-0^{*}$ & $570 / 5 \cdot 7^{*}$ & $570 / 6 \cdot 2^{*}$ \\
\hline$W 121-4^{*}$ & (late) & $0-027$ & 0.709 & $0-251$ & $0-203$ & $0-789$ & 515 & 550 & $550 / 70^{*}$ & $580 / 5 \cdot 2^{*}$ & $<550^{*}$ \\
\hline$W \mid 40-1$ & (rim) & $0-043$ & $0-808$ & $0-132$ & $0-297$ & $0-671$ & 480 & 495 & $540 / 6.4$ & $530 / 3 \cdot 3$ & $550 / 4 \cdot 5$ \\
\hline$W 140-2$ & (rim) & $0-043$ & 0.831 & $0-110$ & $0-310$ & $0-682$ & 430 & 460 & $450 / 6 \cdot 5$ & $480 / 2.6$ & $<550$ \\
\hline
\end{tabular}

- These samples have sillimanite only as an inclusion phase in garnet. Temperature estimates $\left({ }^{\circ} \mathrm{C}\right) \mathrm{T} 1=\mathrm{Perchuk} \& \mathrm{Layrent}$ 'eva $(1983) \mathrm{T} 2$ - Thompson (1976). $P-T$ estimates $\left({ }^{\circ} \mathrm{C} / \mathrm{kb}\right.$ ) based on reaction (11) of text for $P\left(\mathrm{H}_{2} \mathrm{O}\right)=P($ tot) $\mathrm{PT} 3=$ Thompson $(1976) \mathrm{PT} 4=\mathrm{Aranovich} \&$ Podlesskii (1983); PTS = Martignole \& Sisi (1981) (at $\left.\mathrm{nH}_{2} \mathrm{O}=0-8\right)$; calibration of Newton \& Wood (1979) extrapolates to $T<400^{\circ} \mathrm{C}$. All $P-T$ extrapolations below $600^{\circ} \mathrm{C}$ have a large uncertainty. 
TABLE 10

A comparison of cation and isotope geothermometers in banded iron formations

\begin{tabular}{|c|c|c|c|c|c|c|c|c|}
\hline \multirow[b]{2}{*}{ Sample } & \multirow{2}{*}{$\begin{array}{c}\text { Contact } \\
\text { Distance } \\
(m)\end{array}$} & \multirow{2}{*}{$\begin{array}{c}O p x / C p x \\
\mathrm{~T}\left({ }^{\circ} \mathrm{C}\right)\end{array}$} & \multicolumn{2}{|c|}{$G t / O_{p x}$} & \multicolumn{2}{|c|}{$Q z-M t$} & \multicolumn{2}{|c|}{$Q z-O p x$} \\
\hline & & & $\begin{array}{l}\text { core } \\
\mathrm{T}^{\circ}\left({ }^{\circ} \mathrm{C}\right)\end{array}$ & $\underset{\mathrm{T}\left({ }^{\circ} \mathrm{C}\right)}{\mathrm{im}}$ & $\Delta$ & $\mathrm{T}\left({ }^{\circ} \mathrm{C}\right)$ & $\Delta$ & $\mathrm{T}\left({ }^{\circ} \mathrm{C}\right)$ \\
\hline T2-E & $0-1$ & - & 720 & 560 & 9.7 & 530 & 3.8 & 580 \\
\hline$T 2-4$ & $0-1$ & - & 675 & 535 & 9.3 & 550 & 3.6 & 600 \\
\hline $\mathrm{T} 2-10$ & $0-1$ & 580 & - & - & 9.2 & 550 & - & - \\
\hline $\mathrm{T} 7-12$ & $0-1$ & - & 650 & 595 & 96 & 535 & - & - \\
\hline$T 1-4$ & $0-7$ & 530 & - & - & 100 & 520 & - & - \\
\hline T2-9 & 2.4 & 530 & - & - & 7.8 & 620 & - & - \\
\hline$T 2-6$ & $5 \cdot 5$ & - & 680 & 495 & 7.9 & 620 & $2 \cdot 9$ & 700 \\
\hline
\end{tabular}

Contact distance is the distance to the orthogneiss contact. Fractionation factors from Chiba $e t$ al. (1989). Fractionation factor for quartz-diopside is used for $\triangle(Q z-O p x)$.

Temperature estimates from oxygen isotope thermometry in the banded iron formations of the northern Wind River Range are always less than peak temperatures (Sharp et al., 1988). Temperature estimates based on the isotopic fractionations between quartz, magnetite, pyroxene, and garnet generally range from $\sim 600^{\circ} \mathrm{C}$ in the central portion of the large iron formation lenses to $\sim 500^{\circ} \mathrm{C}$ closer to the contact with the surrounding orthogneisses. For all samples, there is a one-to-one correspondence between the preserved fractionations and the degree of deformation that is visible in thin-section. Samples that show more deformation are closer to the orthogneiss contact and preserve lower temperatures. Temperature estimates for the same samples have been made with garnet-orthopyroxene and two-pyroxene thermometry. Additional isotopic measurements have been made with a small-sample laser-based extraction technique (Sharp, 1990). In most cases, cation thermometry yields higher temperatures than oxygen isotope thermometry (Table 10). Furthermore, cation thermometers appear to be less sensitive to minor retrograde deformation. The oxygen isotope fractionations vary systematically with proximity to the surrounding orthogneiss, but these variations are not observed in cation isotope thermometry. Clearly, oxygen isotope thermometry cannot be used to estimate peak temperatures in a high-grade metamorphic terrane that has undergone even minor retrograde deformation. The sensitivity of oxygen isotope thermometry to late-stage deformation can be used to constrain the timing of the deformation, however. If a $P-T-t$ path is obtained by other methods, the timing of late deformation can be determined by oxygen isotope geothermometry.

\section{METAMORPHIC EVOLUTION FOR THE NORTHERN WIND RIVER RANGE}

The higher pressure-temperature data from the garnet inclusions almost certainly predate the lower pressure-temperature data from the matrix minerals. What cannot be determined with the available data is whether the $P-T$ estimates from all assemblages are from the same metamorphic event or two unrelated events. The radiometric data of Aleinikoff et al. (1989) on a granodioritic gneiss indicate that there have been at least two episodes of zircon growth (at 3.2 and $2.7 \mathrm{Ga}$ ). Aleinikoff et al. proposed granulite-facies events at 3.2 and $2.7 \mathrm{Ga}$, with remnants of xenocrystic zircons as old as $3.8 \mathrm{Ga}$. The $3 \cdot 2-\mathrm{Ga}$ event is attributed to highgrade metamorphism based only on zircon morphologies characteristic of high-grade metamorphism, although some grains yielding $3.2 \mathrm{Ga}$ have different morphologies. An alternative explanation for the two sets of dates is that the $3 \cdot 2-\mathrm{Ga}$ zircons may represent the 
formation and emplacement age of the granodiorite, and not that of a high-grade metamorphism. The isotopic data from a lower-grade equivalent metamorphic complex in the Beartooth Mountains, Montana, part of the Wyoming Province, can be used to understand the zircon data from the Wind River Range. $\mathrm{Rb}-\mathrm{Sr}$ and $\mathrm{Sm}-\mathrm{Nd}$ whole-rock ages for a trondhjemitic gneiss component of the Beartooth Mountains are 3.55-3.26 Ga (Mogk et al., 1988), which represents the formation age for the trondhjemite. Rb-Sr whole-rock, $\mathrm{Pb}-\mathrm{Pb}$ whole-rock, and $\mathrm{U}-\mathrm{Pb}$ zircon ages from amphibolites, augen gneisses, granitoids, and granites are clustered at 2.74-2.79 Ga (Mogk et al., 1988; Wooden \& Mueller, 1988), representing the ages of contemporaneous granitic intrusion and metamorphism. Cooling below $400^{\circ} \mathrm{C}$ took place at $2.56 \mathrm{Ga}$, as indicated by a U-Pb sphene date (Mogk et al., 1988). These data are consistent with metamorphism at $2.75 \mathrm{Ga}$ involving a magmatic component and older continental crust $(3 \cdot 2-3 \cdot 4 \mathrm{Ga})$. This would suggest that the zircon data of Aleinikoff et al. (1989) for the Wind River terrane may be interpreted in terms of a protracted metamorphic event $(2.7 \mathrm{Ga})$ involving older continental crust $(3.2 \mathrm{Ga})$. It cannot be ruled out, however, that two metamorphic events occurred, at $3 \cdot 2$ and $2 \cdot 7 \mathrm{Ga}$.

Two explanations are therefore possible for the thermobarometric estimates of this study:

(1) the $P-T$ data from the inclusions within garnet porphyroblasts are recording an early metamorphism $(3.2 \mathrm{Ga})$ and the matrix minerals preserve $P-T$ data from a later metamorphism $(2.7 \mathrm{Ga})$; or

(2) all $P-T$ data are from a single metamorphism $(2.7 \mathrm{Ga})$, with the garnet porphyroblasts recording peak metamorphic conditions and the matrix minerals recording retrograde conditions.

The first possibility requires two separate metamorphic events and the second possibility is compatible with either one or two metamorphisms.

If the $P-T$ data from the inclusions within garnets are recording the first of two metamorphisms, then these data represent the rare preservation of quantitative $P-T$ data from two separate, but similarly high- $P-T$ events $500 \mathrm{Ma}$ apart. Although it is common for granulite-facies terranes to be preserved during subsequent greenschist or amphibolite overprintings, the present study would be very unusual in that a portion of a granulite event survived a later granulite-facies event.

If the calculated pressure-temperature estimates for all assemblages are related to a single, long-lived event, then these data can be combined to constrain the high $P-T-t$ conditions of the northern Wind River Range metamorphism. Although all data are not from the same lithologic units, they are found in close association, both structurally and geographically. It is therefore valid to assume that the various $P-T$ data are from a similar tectonothermal domain and can be combined to constrain the $P-T$ evolution of the northern Wind River Range. Integrating the pressure-temperature estimates of the inclusions within garnet porphyroblasts with those from core and rim matrix minerals results in a clockwise cooling path (Fig. 8). Peak metamorphism occurred at high pressure and temperature $(8 \mathrm{~kb}$ at $800^{\circ} \mathrm{C}$ ), followed by a decompression to $5-6 \mathrm{~kb}$ with only a small temperature decrease. Following this decompression, cooling from 750 to $650^{\circ} \mathrm{C}$ occurred nearly isobarically. These constraints place the Wind River samples within the sillimanite field for a significant portion of their metamorphic history (Fig. 8), consistent with the presence of coarse sillimanite and lack of kyanite.

Combining geothermometry and geobarometry from a group of zoned minerals using independent reactions could yield erroneous results. In this study, $\mathrm{Fe}-\mathrm{Mg}$ exchange thermometry between garnet-orthopyroxene and garnet-clinopyroxene has been combined with garnet-quartz-plagioclase-orthopyroxene geobarometry. The closure temperature for the thermometers is controlled by $\mathrm{Fe}-\mathrm{Mg}$ exchange, and the barometer also involves $\mathrm{Ca}-\mathrm{Al}$ 


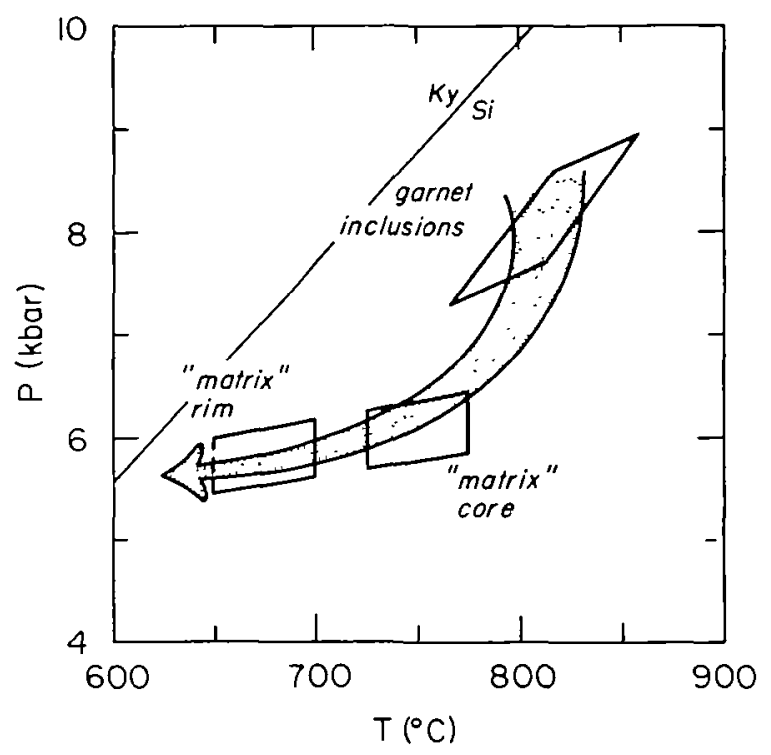

FIG. 8. Combined pressure-temperature estimates from inclusions in garnet and groundmass-matrix minerals. 'Garnet inclusions' are based on mineral equilibria from minerals included in garnet porphyroblasts; 'matrix core' represent pressure-temperature estimates from core compositions of matrix minerals; 'matrix rim' are pressure-temperature estimates based on rum compositions of the matrix minerals. Overall pressure-temperature path is represented by arrow.

exchange (e.g., Bohlen, 1987). If these exchange rates are significantly different, the temperatures and pressures based on rim compositions will not correspond to the same retrograde conditions. Selverstone \& Chamberlain (1990) proposed that the calculated $P-T$ path based on garnet-biotite exchange thermometry and garnet-sillimanitequartz-plagioclase barometry for several terranes yield erroneous isobaric cooling paths. Their principal arguments were that

(1) the retrograde decompression reaction of garnet-sillimanite-quartz $=$ cordierite is commonly observed, and

(2) the feldspars are not zoned.

In the Wind River Range, there is no evidence of decompression reactions. In any event, the presence of decompression reactions does not eliminate the possibility of isobaric cooling, as long as the isobaric cooling occurred below the pressure of the decompression reaction. Lack of Ca-zoning in feldspar and garnet could indeed be due to far slower exchange rates than for Fe-Mg between garnet-pyroxene. The feldspar in the Wind River Range is zoned (Fig. 5), and substantial Ca-zoning in garnets is observed in the Wind River samples and other granulite terranes (Bohlen, 1987), suggesting that Ca exchange does occur. The absolute closure temperatures for the different thermometers and barometers cannot be determined, but even if a higher closure temperature is assumed for the geobarometers than for the geothermometers, the resulting cooling path is still isobaric.

\section{TECTONIC IMPLICATIONS}

Various models based on petrologically constrained $P-T$ paths (e.g., Thompson \& England, 1984; Bohlen, 1987) and numerical thermal calculations (e.g., England \& Richard- 
son, 1977; Wells, 1980; England \& Thompson, 1984) have been proposed to explain mediumto high-grade metamorphism. Although a great many factors can contribute to the thermal history of a given terrane, England \& Thompson (1984) suggested that the major processes that control the pressure-temperature trajectory of a terrane are total heat supply, rate and duration of thickening and subsequent erosion, and the contribution of magmatic heat. Heating of metamorphic terranes occurs by either

(1) deep burial associated with tectonic thickening (England \& Richardson, 1977; England \& Thompson, 1984) or

(2) magmatic underplating and intrusion (Wells, 1980; Bohlen, 1987).

In the first case, pressure will increase substantially during early thickening, followed by a temperature increase resulting from a combination of a relaxation of the geothermal gradient and heat advection controlled by partial melting. Pressures will then decrease during erosion until isostatic equilibrium is achieved. Additional cooling may occur nearly isobarically. A clockwise $P-T$ path is followed. In the second case, early underplating causes rapid heating, followed by increasing pressure after passage of magmas through the terrane. A counterclockwise $P-T$ path will result. In both cases, the final cooling will occur with only a moderate pressure decrease. It is the prograde conditions that must be constrained to distinguish these two possible scenarios.

In most high-grade metamorphic terranes, evidence of the early part of the metamorphic history of a terrane is lost by subsequent re-equilibration at peak $P-T$ or during the early retrograde stages of metamorphism. As a result, there is insufficient evidence to dismiss either of the models presented above. The thermobarometric data from the Wind River Range, if taken as a single event, cover a large enough $P-T$ range to identify the cooling path followed by this terrane. The clockwise cooling path that is obtained is consistent with a tectonic regime of crustal thickening. One expected feature of granulites formed by double thickening is the generation of felsic magmas in the lower plate. These magmas are characteristically absent from regional granulite terranes (Bohlen, 1987), but are abundant in the northern Wind River Range.

Additional tectonic constraints can be placed on the Wind River Range through correlations with lower-grade equivalents of the Wyoming Province. Mogk et al. (1988) concluded that the Northern Snowy Block in the Beartooth Mountains, Montana, underwent oceanic subduction along continental margins and eventual continentalcontinental collision. The Northern Snowy Block and the northern Wind River Range have remarkably similar lithologies and isotopic ages, and it is likely that they are both part of the same orogen. The Wind River Range would therefore represent a higher-temperature, more dismembered equivalent of the Northern Snowy Block. The clockwise $P-T$ path for the granulite-facies Wind River Range is compatible with the tectonic scenario presented by Mogk et al. (1988) for the lower-grade Northern Snowy Block. It appears that the Wyoming Province has undergone at least one large-scale Archean orogeny that may be correlated between widely spaced present-day exposures. Further constraints on the timing and duration of the orogenic events may be possible by combining $\mathrm{U}-\mathrm{Pb}$ dates from zircons and from metamorphic garnets (Mezger et al., 1989) with ${ }^{40} \mathrm{Ar} /{ }^{39} \mathrm{Ar}$ dates from hornblendes and biotites.

\section{DISCUSSION}

Distinctly different temperature and pressure estimates are obtained from the different systems investigated in this study. The highest pressures and temperatures are obtained from mineral inclusions within zoned garnet porphyroblasts. Lower pressures and temperatures 
are preserved in the groundmass chemistries and, in general, the lowest temperatures are obtained with oxygen isotope fractionations. The oxygen isotope thermometer is also the most sensitive thermometer to deformationally induced resetting.

Compositions of mineral inclusions within zoned garnet porphyroblasts have previously been used to estimate prograde metamorphic conditions in greenschist and amphibolite terranes (e.g., Thompson et al., 1977; Tracy, 1982; Selverstone et al., 1984; St-Onge, 1984, 1987; Hodges \& Silverberg, 1988), but much less attention has been paid to granulite terranes. Inclusions in garnets may be the best source for determining prograde or peak conditions in granulite-facies rocks. Garnet growth following the equilibration of the mineral inclusions will retard further reaction and allow for preservation of chemical information pertaining to the highest grades of metamorphism. The groundmass assemblages do not preserve the very high temperatures associated with the mineral inclusions in the garnets. Even the cores of mineral grains appear to have undergone some diffusional retrograde exchange. Other granulite terranes do preserve higher pressures and temperatures (Bohlen, 1987). Different tectonic regimes and prograde $P-T$ paths are proposed for these terranes. A common feature to both the Wind River terrane and those presented by Bohlen is that the peak preserved pressures and temperatures are those that existed immediately before the onset of isobaric cooling. The Wind River terrane passed through the peak $P-T$ field presented by Bohlen for other granulite-facies terranes, but unlike these terranes, underwent decompression before isobaric cooling. If this decompression was a long-lived process, then the observed groundmass resetting would be expected.

A present limitation of metamorphic petrology is the lack of a thorough understanding of the diffusional exchange processes and rates that occur during retrogression. Although this has hampered efforts to determine peak pressures and temperatures for high-grade metamorphic terranes, it is precisely this partial resetting of chemical systems that allows the more dynamic variations in pressure and temperature to be determined. As further quantifications are made on rates of solid-solid reactions, diffusion, and the effects of deformation and fluid presence on these rates, better determinations of $P-T-t$ paths will be made.

\section{ACKNOWLEDGEMENTS}

This work was supported in part by NSF grant EAR-84-08168 and EAR-82-12764 to E.J.E., GSA grants $3219-83$ and 349485 to Z.D.S., and Grant-in-Aid of Research from Sigma Xi to Z.D.S. The authors would like to express their thanks to J. N. Aleinikoff for an early preprint of his paper, and especially to M. A. Cosca and G. R. Helffrich for their help in the field. The reviews of K. Mezger, J. Morrison, and M. R. St-Onge, and the editorial handling and comments of $\mathrm{D}$. Rumble, are greatly appreciated and improved the manuscript.

\section{REFERENCES}

Aleinikoft, J. N., Williams, I. S, Compston, W., Stuckless, J. S, \& Worl, R. G., 1989. Evidence for an early Archean component in the middle to late Archean gneisses of the Wind River Range, west-central Wyoming: conventional and ion microprobe U-Pb data. Contr. Miner. Petrol. 101, 198-206.

Anovitz, L. M., \& Essene, E. J, 1987. Compatibility of geobarometers in the system $\mathrm{CaO}-\mathrm{FeO}-\mathrm{Al}_{2} \mathrm{O}_{3}-\mathrm{SiO}_{2}-\mathrm{TiO}_{2}$ : implications for garnet mixing models. J. Geol. 95, 633-45.

— Treiman, A. H., Essene, E. J., Hemingway, B. S., Westrum, E. F, Jr, Wall, V. J., Burriel, R., \& Bohlen, S. R., 1985. The heat-capacity of ilmenite and phase equilibria in the system Fe-Ti-O. Geochim. Cosmochim. Acta 49, $2027-40$.

Aranovich, L. Ya., \& Podlesskii, K. K, 1983. The cordierite-garnet-sillimanite-quartz equilibrium: experiments and applications. In: Saxena, S. K. (ed.) Advances in Physical Geochemistry, 3. Heidelberg: Springer-Verlag, 173-97. 
Baker, C. L, 1946. Geology of the northwestern Wind River Mountains, Wyoming. Geol. Soc. Am. Bull. 57, 565-96. Bassett, W. A., \& Giletti, B. J., 1963. Precambrian ages in the Wind River Mountains, Wyoming. Ibid. 74, $209-12$.

Bayley, R. W., 1963. A preliminary report on the Precambrian iron deposits near Atlantic City, Wyoming. $U . S$. Geol. Surv. Bull. 1142C, 39 pp.

1965a. Geologic map of the Louis Lake quadrangle, Fremont County, Wyoming. U.S. Geol. Surv. Quad. Map GQ-461.

- 1965b. Geologic map of the South Pass City quadrangle, Fremont County, Wyoming. Ibid. GQ-458.

- Proctor, P. D., \& Condie, K. C., 1973. Geology of the South Pass area, Fremont County, Wyoming. U.S. Geol. Suro. Prof. Paper 793, 39 pp.

Berg, R. R., 1962. Mountain flank thrusting in Rocky Mountain foreland, Wyoming and Colorado. Bull. Am. Assoc. Petrol. Geol. 48, 1019-32.

- Romberg, F. E., 1966. Gravity profile across the Wind River Mountains, Wyoming. Geol. Soc. Am. Bull. 77, 647-56.

Berger, G. W., \& York, D, 1981. Geothermometry from ${ }^{40} \mathrm{Ar} /{ }^{39}$ Ar dating experiments. Geochim. Cosmochim. Acta 45, 795-811.

Birch, F., 1966. Compressibility: elastic constants. In: Clark, S. P., Jr (ed.) Handbook of Physical Constants. Geol. Soc. Am. Mem. 97, 97-173.

Bohlen, S. R., 1987. Pressure-tempecature-time paths and a tectonic model for the evolution of granulites. J. Geol. 95, 617-32.

- Boettcher, A. L., 1981. Experimental investigations and geological applications of orthopyroxene geobarometry. Am. Miner. 66, 951-64.

- Dollase, W. A., \& Wall, V. J., 1986. Calibration and application of spinel equilibria in the system $\mathrm{FeO}-\mathrm{Al}_{2} \mathrm{O}_{3}-\mathrm{SiO}_{2}$. J. Petrology 27, 1143-56.

-Essene, E. J., \& Boettcher, A. L., 1980. Reinvestigation and application of olivine-quartz-orthopyroxene barometry. Earth Planet. Sci. Lett. 47, 1-10.

Wall, V. J., \& Boettcher, A. L., 1983a. Experimental investigations and geologic applications of equilibria in the system $\mathrm{FeO}-\mathrm{TiO}_{2}-\mathrm{Al}_{2} \mathrm{O}_{3}-\mathrm{SiO}_{2}-\mathrm{H}_{2} \mathrm{O}$. Am. Miner. 68, 1049-58.

-Wall, V. J., \& Boettcher, A. L., 1983b. Geobarometry in granulites. In: Saxena, S. K. (ed.) Advances in Physical Geochemistry, 3. Heidelberg: Springer-Verlag, 141-72.

- Metz, G. W, Essene, E. J, Anovitz, L. M., Westrum, E. F., \& Wall, V. J., 1983c. Thermodynamics and phase equilibrium of ferrosilite: potential oxygen barometer in mantle rocks. Trans. Am. Geophys. Union 64, 350.

Brace, W. F., Scholz, C. H, \& La Mori, P. N., 1969. Isothermal compressibility of kyanite, andalusite, and sillimanite from synthetic aggregates. J. Geophys. Res. 74, $2089-98$.

Branson, E. B., \& Branson, C. C, 1941. Geology of the Wind River Mountains. Bull. Am. Assoc. Petrol. Geol. 25, 120-51.

Cheang, K. K., Wenner, D. B., \& Stuckless, J. S., 1985. Oxygen isotope constraints on the origin of Precambrian granites from the southern Wind River Range and the Granite Mountains, central Wyoming. U.S. Geol. Surv. Bull. 1622, Chapters A-T, Shorter Contributions to Isotope Geology, 109-29.

Chiba, H., Chacko, T., Clayton, R. N., \& Goldsmith, J. R., 1989. Oxygen isotope fractionations involving diopside, forsterite, magnetite, and calcite: application to geothermometry. Geochim. Cosmochim. Acta 53, $2985-95$.

Chopin, C., 1984. Coesite and pure pyrope in high-grade blueschists of the Western Alps: a first record and some consequences. Contr. Miner. Petrol. 86, 107-18.

Condie, K. C., 1976. The Wyoming Archean Province in the western United States, In: Windley, B. F. (ed.) The Earth's Early History. New York: John Wiley, 499-510.

— Leech, A. P., \& Baadsgaard, H., 1969. Potassium-argon ages of Precambrian mafic dikes in Wyoming Geol. Soc. Am. Bull. 80, 899-906.

Cosca, M. A. Sutter, J. F., \& Essene, E. J., in press. Late metamorphic cooling and inferred uplift/erosion history of the Ontario Grenville Province: constraints from ${ }^{40} \mathrm{Ar} /{ }^{39} \mathrm{Ar}$ thermochronology. Tectonics.

Crank, J., 1975. The Mathematics of Diffusion. Oxford: Oxford University Press, $414 \mathrm{pp}$.

Cygan, R. T., \& Lasaga, A. C., 1985. Self-diffusion of magnesium in garnet at $750^{\circ} \mathrm{C}$ to $900^{\circ} \mathrm{C}$. Am. J. Sci. 285 , 328-50.

Davidson, P. M., \& Lindsley, D. H., 1985. Thermodynamic analysis of quadrilateral pyroxenes. Part II. Model calibration from experiments and applications to geothermometry. Contr. Miner. Petrol. 91, 390-404.

Dodson, M. H., 1973. Closure temperature in cooling geochronological and petrological systems. Ibid. 40, $259-74$.

Ellis, D. J., \& Green, D. H., 1979. An experimental study of the effect of Ca upon garnet-clinopyroxene exchange equilibria. J. Petrology 26, 633-62.

England, P. C., \& Richardson, S. W., 1977. The influence of erosion upon the mineral facies of rocks from different metamorphic environments. J. Geol. Soc. Lond. 134, 201-13.

-Thompson, A. B., 1984. Pressure-temperature-time paths of regional metamorphism. I. Heat transfer during the evolution of regions of thickened continental crust. J. Petrology 25, 894-928.

Essene, E. J., 1982. Geologic thermometry and barometry. In: Ferry, J. M. (ed.) Characterization of Metamorphism through Mineral Equilibria. Miner. Soc. Am., Rev. Miner. 10, 153-206.

1989. The current status of thermobarometry in metamorphic rocks. In: Daly, J. S., Cliff, R. A., \& Yardley, B. W. D. (eds.) Evolution of Metamorphic Belts. Geol. Soc. Spec. Paper 43, 1-44.

Ganguly, J., \& Saxena, S. K, 1984. Mixing properties of aluminosilicate garnets: constraints from natural and 
experimental data and applications to geothermo-barometry. Am. Miner. 69, 88-97.

Granger, H. C., McKay, E. J., Mattick, R. E., Patten, L. L. \& MacIlroy, P., 1971. Mineral resources of the Glacier Primitive Area, Wyoming. U.S. Geol. Suro. Bull. 1319-F, 113 pp.

Hazen, R. M., 1976. Effects of temperature and pressure on the crystal structure of forsterite. Am. Miner. 61, 1280-93.

- Finger, L. W., 1981. Bulk moduli and high-pressure crystal structures of rutile-type compounds. J.Phys. Chem. Solids 42, 143-51.

Hodgc, D. S., \& Worl, R. G., 1965. Multiple metamorphic episodes in Precambrian rocks near South Pass, Wyoming. Contr. Geol. Univ. Wyoming 4, 51-7.

Hodges, K. V, \& Silverberg, D. S., 1988. Thermal evolution of the greater Himalaya, Garhwal, India. Tectonics 7, $583-600$.

Koesterer, M. E., Frost, C. D., Frost, B. R., Hulsebosch, T. P., Bndgwater, D., \& Worl, R. G., 1987. Development of the Archean crust in the Medina Mountain area, Wind River Range, Wyoming (U.S.A.). Precambrian Res. 37, 287-304.

Lee, H. Y., \& Ganguly, J., 1987. Equilibrium compositions of coexisting garnet and orthopyroxene: experimental determinations in the system $\mathrm{FeO}-\mathrm{MgO}-\mathrm{Al}_{2} \mathrm{O}_{3}-\mathrm{SiO}_{2}$, and applications. J. Petrology 29, 93-113.

Lindsley, D. H., 1983. Pyroxene thermometry. Am. Miner. 68, 477-93.

Link, P. K., Anderson, I. C., Hengesh, J. V., \& Worl, R. G., 1985. Geologic map of the Horseshoe Lake quadrangle, Sublette County, Wyoming. U.S. Geol. Surv. Open-File Rep. 85-0449.

Martignole, J, \& Sisi, J-C., 1981. Cordierite-garnet- $\mathrm{H}_{2} \mathrm{O}$ equilibrium: a geological thermometer, barometer and water fugacity indicator. Contr. Miner. Petrol. 77, 38-46.

Meagher, E. P., \& Lager, G. A, 1979. Polyhedral thermal expansion in the $\mathrm{TiO}_{2}$ polymorphs: refinement of the crystal structures of rutile and brookite at high temperature. Can. Miner. 17, 77-85.

Metz, G. W., Anovitz, L. M., Essene, E. J., Bohlen, S. R., \& Westrum, E. F., Jr, 1983. The heat capacity and phase equilibria of almandine. Trans. Am. Geophys. Union 64, 346.

Mezger, K., Hanson, G. N., \& Bohlen, S. R., 1989. U-Pb systematics of garnet: dating the growth of garnet in the Late Archean Pikwitonei granulite domain at Cauchon and Natawahunan Lakes, Manitoba, Canada. Contr. Miner. Petrol. 101, 136-48.

Mitra, G., \& Frost, R. B., 1981. Mechanisms of deformation within Laramide and Precambrian deformation zones in basement rocks of the Wind River Mountains. In: Boyd, D. W. (ed.) Rocky Mountain Foreland Basement Tectonics. Contr. Geol., Univ. Wyoming 19, 161-73.

Mogk, D. W., Mueller, P. A., \& Wooden, J. L., 1988. Archean tectonics of the North Snowy Block, Beartooth Mountains, Montana. J. Geol. 69, 125-41.

Naylor, R. S., Steiger, R. H., \& Wasserburg, G. J., 1970. U-Th-Pb and Rb-Sr systematics in a $2700 \times 10^{6}$-year old pluton from the southern Wind River Range, Wyoming. Geochim. Cosmochim. Acta 34, 1133-59.

Newton, R. C., 1972. An experimental determination of the high-pressure stability limits of magnesian cordierite under wet and dry conditions. J. Geol. 80, 398-420.

Charlu, T. V., \& Kleppa, O. J., 1980. Thermochemistry of the high structural state plagioclases. Geochim. Cosmochim. Acta 44, 933-41.

Wood, B. J., 1979. Thermodynamics of water in cordierite and some petrologic consequences of cordierite as a hydrous phase. Contr. Miner. Petrol. 68, 391-405.

Oftendahl, C, 1953. Petrologic reconnaissance in the Precambrian of the western part of the Wind River Mountains, Wyoming. Norsk Geol. Tidsskrift 32, 1-17.

Pattison, D. R. M., \& Newton, R. C., 1989. Reversed experimental calibration of the garnet-clinopyroxene Fe-Mg exchange thermometer. Contr. Miner. Petrol. 101, 87-103.

Pearson, R. C, Kilsgaard, T. H., \& Patton, L. L., 1971. Mineral resources of the Popo Agie Primitive Area, Fremont and Sublette Counties, Wyoming. US. Geol. Surv. Bull. 1353-B, 55 pp.

Perchuk, L. L., \& Lavrent'eva, 1. V. 1983. Experimental investigation of exchange equilibria in the system cordierite-gamet-biotite. In: Saxena, S. K. (ed.) Advances in Physical Geochemistry, 3. Heidelberg: SpringerVerlag, 199-239.

Perry, K., Jr., 1965. High-grade regional metamorphism of Precambrian gneisses and associated rocks, Paradise Basin Quadrangle, Wind River Mountains, Wyoming. Ph.D. Thesis, Yale University, New Haven, CT.

Robie, R. A., Finch, C. B., \& Hemingway, B. S. 1982. Heat capacity and entropy of fayalite $\left(\mathrm{Fe}_{2} \mathrm{SiO}_{4}\right)$ between $5 \cdot 1$ and $383 \mathrm{~K}$ : comparison of calorimetric and equilibrium values for the QFM buffer reaction. Am. Miner. 67, 463-69.

- Hemingway, B. S., 1984. Entropies of kyanite, andalusite, and sillimanite: additional constraints on the pressure of the $\mathrm{Al}_{2} \mathrm{SiO}$, triple point. Ibid. 69, 298-306.

Fisher, J. R., 1978. Thermodynamic properties of minerals and related substances at $298.15 \mathrm{~K}$ and 1 bar $\left(10^{5}\right.$ pascals) pressure and at higher temperatures. U.S. Geol. Surv. Bull. 1452, $456 \mathrm{pp}$., reprinted with corrections.

Robinson, G. R., Jr, Haas, J. L., Jr, Schafer, C. M, \& Haselton, H. T., Jr, 1982. Thermodynamic and thermophysical properties of selected phases in the $\mathrm{MgO}_{-} \mathrm{SiO}_{2}-\mathrm{H}_{2} \mathrm{O}-\mathrm{CO}_{2}, \mathrm{CaO}-\mathrm{Al}_{2} \mathrm{O}_{3}-\mathrm{SiO}_{2}-\mathrm{H}_{2} \mathrm{O}-\mathrm{CO}_{2}, \mathrm{Fe}-\mathrm{FeO}_{-}-\mathrm{Fe}_{2} \mathrm{O}-$ $\mathrm{SiO}_{2}$, chemical systems with special emphasis on the properties of basalts and their mineral component. U.S. Geol. Suro. Open-File Rep. 83-79, 429 pp.

Rosenfeld, J L., 1970. Rotated garnets in metamorphic rocks. Geol. Soc. Am. Spec. Paper 129,105 pp. 
Selverstone, J., \& Chamberlain, C. P., 1990. Apparent isobaric cooling paths from granulites: two counterexamples from British Columbia and New Hampshire. Geology 18, 307-10.

- Spear, F. S., Franz, G, \& Morteani, G., 1984. High-pressure metamorphism in the SW Tauern Window, Austria: $P-T$ paths from bornblende-kyanite-staurolite schists. J. Petrology 25, 501-31.

Sharp, Z. D., 1988. Metamorphism and oxygen isotope geochemistry of the Northern Wind River Range, Wyoming. Ph.D. Thesis, University of Michigan, Ann Arbor, MI.

- 1990. A laser-based microanalytical method for the in situ determination of oxygen isotope ratios of silicates and oxides. Geochim. Cosmochim. Acta 54, 1353-7.

- Essene, E. J., 1984. A low pressure granulite facies taconite: implications for Archean heat flow. Geol. Soc. Am. Abstr. Prog. 16, 651.

- O'Neil, J. R., \& Essene, E. J., 1988. Oxygen isotope variations in granulite-grade iron formations: constraints on oxygen diffusion and retrograde isotopic exchange. Contr. Miner. Petrol. 98, 490-501.

Smithson, S. B., Brewer, J. A., Kaufman, S., Oliver, J. E., \& Zawislak, R. L., 1980. Complex Archean lower crustal structure revealed by COCORP crustal reflection profiling in the Wind River Range, Wyoming. Earth Planet. Sci. Lett. 46, 295-305.

- Ebens, R. J., 1971. Interpretation of data from a 305-kilometer borehole in Precambrian crystalline rocks, Wind River Mountains, Wyoming. J. Geophys. Res. 76, 7079-87.

Spall, H., 1971. Paleomagnetism and K-Ar age of mafic dikes from the Wind River Range, Wyoming. Geol. Soc. Am. Bull. 82, 2457-72.

Spear, F. S., \& Rumble, D, III, 1986. Pressure, temperature, and structural evolution of the Orfordville Belt, West-Central New Hampshire. J. Petrology 27, 1071-93.

- Selverstone, J., 1983. Quantitative $\boldsymbol{P}-T$ paths from zoned minerals: theory and tectonic applications. Contr. Miner. Petrol. 83, 348-57.

St-Onge, M. R., 1984. Geothermometry and geobarometry in pelitic rocks of north-central Wopmay Orogen (early Proterozoic, Northwest Territories, Canada. Geol. Soc. Am. Bull. 95, 196-208.

- 1987. Zoned poikiloblastic garnets: $P-T$ paths and synmetamorphic uplift through $30 \mathrm{~km}$ of structural depth, Wopmay Orogen, Canada. J. Petrology 28, 1-21.

Stuckless, J. S., Hedge, C. E., Worl, R. G., Simmons, K. R., Nkomo, I. T., \& Wenner, D. B., 1986. Isotopic studies of the late Archean plutonic rocks of the Wind River Range, Wyoming. Geol. Soc. Am. Bull. 96, 850-60.

Sykes, J. A., 1985. High grade metamorphism of iron-rich bodies in Archean gneiss, Wind River Mountains, Wyoming. Unpublished M.S. Thesis, University of Minnesota.

Thompson, A. B., 1976. Mineral reactions in pelitic rocks: II. Calculation of some $P-T-X(\mathrm{Fe}-\mathrm{Mg})$ phase relations. Am. J. Sci. 276, 425-54.

- England, P. C., 1984. Pressure-temperature-time paths of regional metamorphism II. Their inference and interpretation using mineral assemblages in metamorphic rocks. J. Petrology 25, 929-55.

- Tracy, R. J., Lyttle, P. T., \& Thompson, J. B., Jr, 1977. Prograde reaction histories deduced from compositional zonation and mineral inclusions in garnet from the Gassetts schist, Vermont. Am. J. Sci. 277, 1152-67.

Tracy, R. J., 1982. Compositional zoning and inclusions in metamorphic minerals. In: Ferry, J. M. (ed.) Characterization of Metamorphism through Mineral Equilibria. Miner. Soc. Am. Rev. Miner. 10, 355-97.

Wells, P. R. A., 1980. Thermal models for the magmatic accretion and subsequent metamorphism of continental crust. Earth Planet. Sci. Lett. 46, 253-65.

Winter, J. K., \& Ghose, S., 1979. Thermal expansion and high-temperature crystal chemistry of the $\mathrm{Al}_{2} \mathrm{SiO}_{3}$ polymorphs. Am. Miner. 64, 573-86.

Wooden, J. L, \& Mueller, P. A., 1988. Pb, $\mathrm{Sr}$, and $\mathrm{Nd}$ isotopic compositions of a suite of Late Archean, igneous rocks, eastern Beartooth Mountains: implications for crust-mantle evolution. Earth Planet. Sci. Lett. 87, $59-72$.

Worl, R. G., 1968. Taconite in the Wind River Mountains, Sublette County, Wyoming. U.S. Geol. Surv. Prelim. Rep. 10.

- Lee, G. K., \& Long, C. L., 1984. Mineral resource potential of the Bridger Wilderness and the Green-Sweetwater roadless area, Sublette and Fremont Counties, Wyoming. U.S. Geol. Surv. Misc. Field Studies Map 1636-A. 